\title{
Coarse-grained model of mitochondrial metabolism enables subcellular flux inference from fluorescence lifetime imaging of NADH
}

\author{
Xingbo Yang ${ }^{1}$ and Daniel J. Needleman ${ }^{1,2}$ \\ ${ }^{1}$ Department of Molecular and Cellular Biology and John A. Paulson School of Engineering and \\ Applied Sciences, Harvard University, Cambridge, MA 02138. ${ }^{2}$ Center for Computational \\ Biology, Flatiron Institute, New York, United States
}

\begin{abstract}
Mitochondrial metabolism is of central importance to diverse aspects of cell and developmental biology. Defects in mitochondria are associated with many diseases, including cancer, neuropathology, and infertility. Our understanding of mitochondrial metabolism in situ and dysfunction in diseases are limited by the lack of techniques to measure mitochondrial metabolic fluxes with sufficient spatiotemporal resolution. Herein, we develop a new method to infer mitochondrial metabolic fluxes in living cells with subcellular resolution from fluorescence lifetime imaging of NADH. This result is based on the use of a coarse-grained model of mitochondrial metabolism. We test the model in mouse oocytes subject to a wide variety of perturbations by comparing predicted fluxes through the electron transport chain to direct measurements of oxygen consumption rate. Using this method, we discover a subcellular spatial gradient of mitochondrial metabolic flux in mouse oocytes. This label-free technique will help provide new insights into the spatiotemporal regulation of metabolic fluxes in developing organisms and in diseases.
\end{abstract}

\section{Introduction}

Decades of extensive work have produced a remarkable body of detailed information about the biochemistry of mitochondrial energy metabolism (Salway, 2017). In brief, metabolites, such as pyruvate, are transported into mitochondria, where they are broken down and their products enter the tricarboxylic acid cycle (TCA). The TCA is composed of a number of chemical reactions, which ultimately reduces $\mathrm{NAD}^{+}$to $\mathrm{NADH}$. NADH and oxygen are then utilized by the electron transport chain (ETC) to pump hydrogen ions across the mitochondrial membrane. ATP synthase uses this proton gradient to power the synthesis of ATP from ADP (Mitchell, 1961). The activities of mitochondrial energy metabolism are characterized by the fluxes through these pathways: i.e. the mitochondrial metabolic fluxes. However, despite the wealth of knowledge concerning mitochondrial biochemistry, it is still unclear what processes set mitochondrial metabolic fluxes in cells (Dumollard et al., 2007; Blerkom, 2011; Yellen 2018) or precisely how these fluxes are misregulated in diseases (Brand and Nicholls, 2011; Lin and Flint Beal, 2006; Wallace, 2012; Bratic and Larsson, 2013; Lowell and Shulman, 2005; Mick et al., 2020). The limitations of current techniques for measuring mitochondrial metabolic fluxes presents a major challenge. In particular, there is a lack of techniques to measure mitochondrial metabolic fluxes with subcellular spatial resolution. Bulk biochemical techniques, such as measures of oxygen consumption and nutrient uptake rates (Ferrick et al., 2008; Houghton et al., 1996; Lopes et al., 2005), and isotope tracing 
by mass spectrometry (Wiechert, 2001), require averaging over large populations of cells. Such techniques cannot resolve cellular, or subcellular, metabolic heterogeneity (Takhaveev and Heinemann, 2018; Aryaman et al., 2019). Biochemical approaches for measuring mitochondrial metabolic fluxes are also often destructive (Wiechert, 2001; Saks, et al 1998), and thus cannot be used to observe continual changes in fluxes over time. Fluorescence microscopy provides a powerful means to measure cellular and subcellular metabolic heterogeneity continuously and nondestructively, with high spatiotemporal resolution. However, while fluorescent probes can be used to measure mitochondrial membrane potential (Perry et al., 2011) and the concentration of key metabolites (Imamura et al., 2009; Berg et al., 2009; Díaz-García et al., 2017; Martin et al., 2014), it is not clear how to relate those observables to mitochondrial metabolic fluxes.

Endogenous NADH has long been used to non-invasively probe cellular metabolism because $\mathrm{NADH}$ is autofluorescent, while $\mathrm{NAD}^{+}$is not (Heikal, 2010). Fluorescence lifetime imaging microscopy (FLIM) of NADH autofluorescence allows quantitative measurements of the concentration of $\mathrm{NADH}$, the fluorescence lifetimes of $\mathrm{NADH}$, and the fraction of NADH molecules bound to enzymes (Becker, 2012; Becker, 2019; Bird et al., 2005; Skala et al., 2007; Heikal, 2010; Sharick et al., 2018; Sanchez et al., 2018; Sanchez et al., 2019; Ma et al., 2019). It has been observed that the fraction of enzyme-bound NADH and NADH fluorescence lifetimes are correlated with the activity of oxidative phosphorylation, indicating that there is a connection between NADH enzyme-binding and mitochondrial metabolic fluxes (Bird et al., 2005; Skala et al., 2007). The mechanistic basis of this empirical correlation has been unclear. Here we show, that if properly interpreted, FLIM of NADH can be used to quantitatively measure flux through the mitochondrial electron transport chain, and thus provides a means to measure mitochondrial metabolic fluxes. Our results are based on a general, coarse-grained NADH redox model. We validate this model in mouse oocytes subject to a range of perturbations by comparing predicted fluxes from FLIM to direct measurements of the rate of oxygen consumption, and by selfconsistency criterion. Using this method, we discovered a subcellular spatial gradient of mitochondrial metabolic fluxes in mouse oocytes. Thus, FLIM of NADH can be used to noninvasively and continuously measure mitochondrial metabolic fluxes with subcellular resolution.

We used meiosis II arrested mouse oocytes as a model system to study mitochondrial metabolism with FLIM of NADH. ATP synthesis in mouse oocytes occurs primarily through oxidative phosphorylation using pyruvate, without an appreciable contribution from glycolysis (Houghton et al., 1996). Mouse oocyte can be cultured in vitro using chemically well-defined media (Biggers and Racowsky, 2002). They can directly take up pyruvate supplied to them or derive it from lactate through the activity of lactate dehydrogenase (LDH) (Lane and Gardner, 2000), and they can remain in a steady state for hours with constant metabolic fluxes. While NADH and NADPH are difficult to distinguish with fluorescence measurements, the concentration of NADH in mouse oocytes is 40 times greater than the concentration of NADPH (Bustamante et al., 2017), so the autofluorescence signal from these cells can be safely assumed to result from NADH. 


\section{Results}

\section{Quantifying mitochondrial metabolism as a function of oxygen level using FLIM of NADH}

To investigate the impact of quantitatively perturbing mitochondria, we continually varied the concentration of oxygen in the media, from $50 \pm 2 \mu \mathrm{M}$ to $0.26 \pm 0.04 \mu \mathrm{M}$, while imaging NADH autofluorescence of oocytes with FLIM (Figure 1a, top, black curve; Video 1). We used a machinelearning based algorithm to segment mitochondria in the obtained images (Berg et al., 2019) (Figure $1 \mathrm{~b}$ and Figure 1-figure supplement 1), allowing us to specifically study the response of NADH in mitochondria. The intensity of NADH in mitochondria, $I$, increased with decreasing oxygen concentration (Figure 1a, top, red), as is readily seen from the raw images (Figure 1a, middle). Restoring oxygen to its original level caused a recovery of NADH intensity, indicating that the observed changes are reversible (Figure 1a; Video 1). We next grouped all detected photons from mitochondria to form histograms of photon arrival times from NADH autofluorescence for each time point (Figure 1a. lower). We fit the histograms using a model in which the NADH fluorescence decay, $F(\tau)$, is described by the sum of two exponentials, $F(\tau)=f \cdot \exp \left(-\frac{\tau}{\tau_{l}}\right)+(1-f) \cdot \exp \left(-\frac{\tau}{\tau_{s}}\right)$, where $\tau_{l}$ and $\tau_{s}$ are long and short fluorescence lifetimes, corresponding to enzyme-bound NADH and freely-diffusing NADH, respectively, and $f$ is the fraction of enzyme-bound NADH (Sanchez et al., 2018; Sanchez et al., 2019) (Methods).

We repeated the oxygen drop experiments for a total of 68 oocytes. We approximated the slow decrease in oxygen levels as quasistatic, and averaged data from all oocytes to determine how mitochondrial NADH intensity, $I$, lifetimes, $\tau_{l}$ and $\tau_{s}$, and the fraction of enzyme-bound NADH, $f$, varied with oxygen level (Figure 1c). We next calibrated the measured intensity in oocytes by comparison with measures of known concentrations of NADH in vitro, and used the experimentally measured long and short lifetimes to correct for the difference in molecular brightness between free and bound NADH (Figure 1-figure supplement 2; Figure 1-figure supplement 3), to determine how the concentrations of free NADH, $\left[N A D H_{f}\right]$, and enzyme-bound $\mathrm{NADH},\left[N A D H_{b}\right]$, depend on oxygen level (Figure 1c, lower right). $\left[N A D H_{f}\right]$ increased as oxygen fell below $\sim 10 \mu \mathrm{M}$, while $\left[N A D H_{b}\right]$ did not vary with oxygen level. 


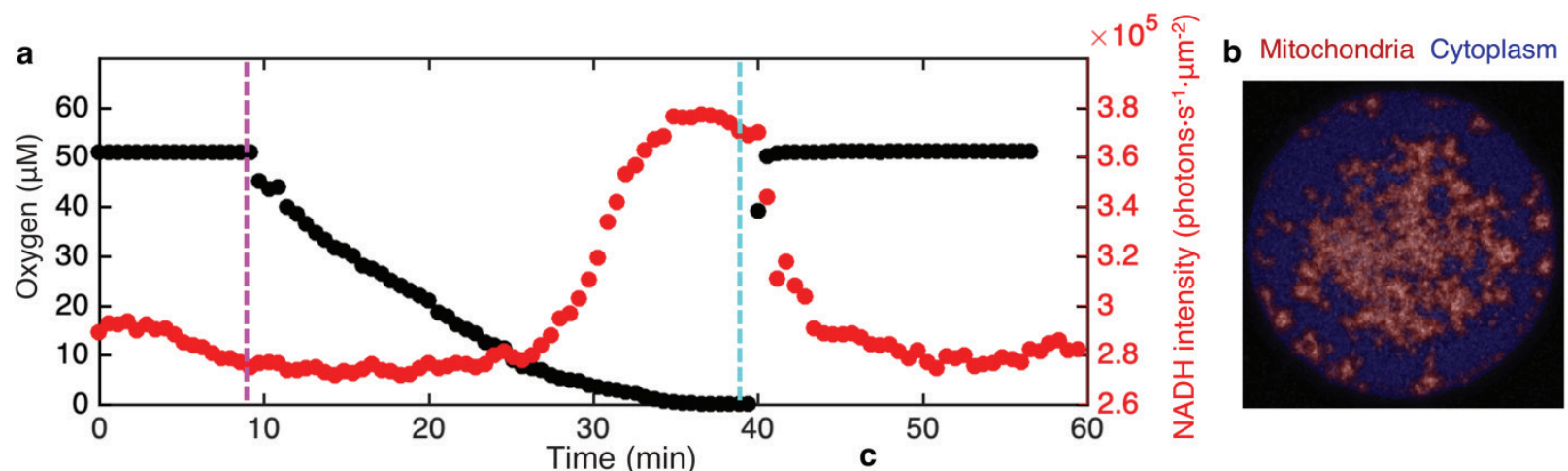

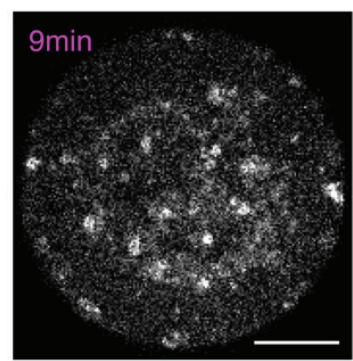

$\times 10^{3}$
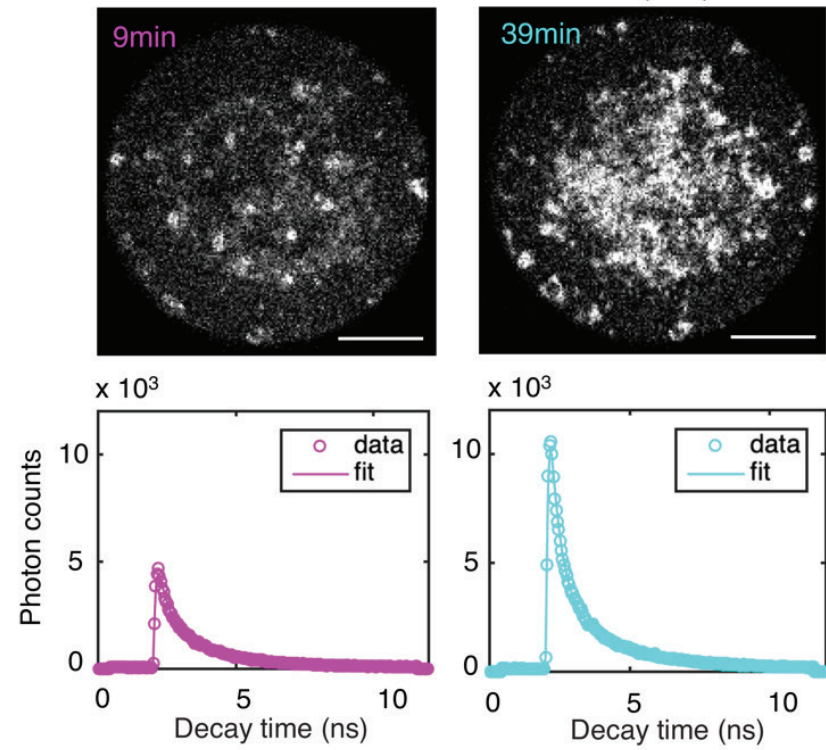

$\times 10^{3}$

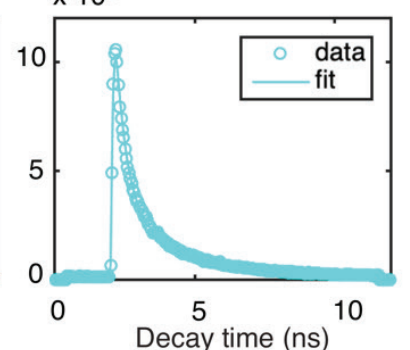

C
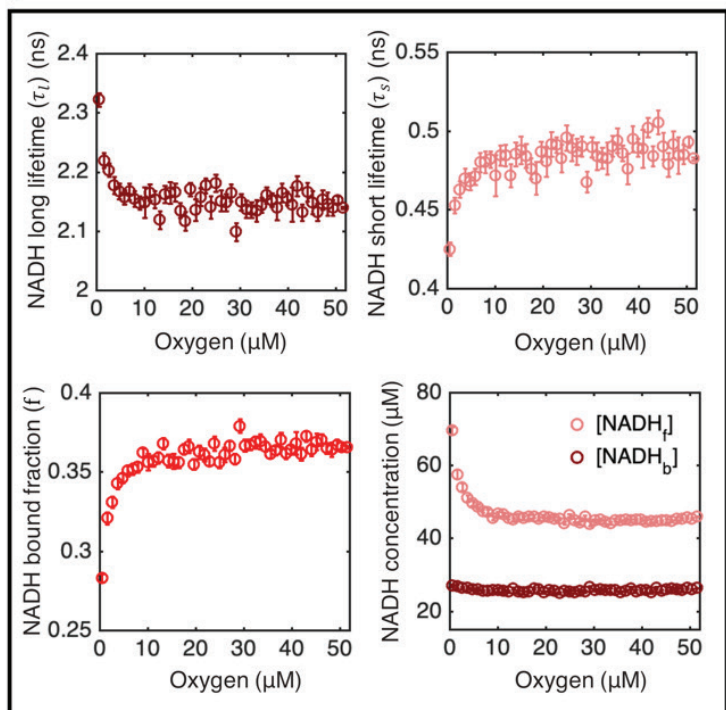

Figure 1 | FLIM measurements of the response of mitochondrial NADH as a function of oxygen level. a, Top row: oxygen level (black circles) and mitochondrial NADH intensity (red circles) as a function of time. Middle row: NADH intensity images of MII mouse oocyte at high and low oxygen levels corresponding to times indicated by the vertical lines. Scale bar, $20 \mu \mathrm{m}$. Bottom row: FLIM decay curves of the corresponding oocyte at low and high oxygen levels, with corresponding fits. b, NADH-intensity-based segmentation of mitochondria and cytoplasm. c, Mitochondrial NADH long fluorescence lifetime $\tau_{l}$ (upper left), short fluorescence lifetime $\tau_{s}$ (upper right), and bound fraction $f$ (lower left) as a function of oxygen level ( $\mathrm{n}=68$ oocytes). These FLIM parameters can be used in combination with intensity, $I$, and proper calibration, to obtain the concentration of free NADH, $\left[N A D H_{f}\right]$, and the concentration of enzyme-bound NADH, $\left[N A D H_{b}\right]$, in mitochondria as a function of oxygen (lower right). Error bars are standard error of the mean (s.e.m).

Video 1 | NADH intensity as a function of oxygen level in mouse oocyte. Left: Imaging of NADH from autofluorescence of mouse oocyte. Right: Real time measurement of oxygen level in the culturing media.

\section{Developing coarse-grained NADH redox model to relate FLIM of NADH to activities of mitochondrial metabolic pathways}

We next developed a mathematical model of NADH redox reactions to relate these quantitative FLIM measurements to activities of mitochondrial metabolic pathways. NADH is a central coenzyme that binds to enzymes and facilitates redox reactions by acting as an electron carrier. There are two categories of enzymes associated with NADH redox reactions, which together form 
a redox cycle: oxidases that oxidize NADH to $\mathrm{NAD}^{+}$and reductases that reduce $\mathrm{NAD}^{+}$to $\mathrm{NADH}$. The major NADH oxidase in mitochondria is complex I of ETC for eukaryotic cells. There are many NADH reductases in mitochondria because NADH can be reduced through different pathways depending on the energy substrate. These pathways include the TCA cycle, fatty acid catabolism via beta oxidation, amino acid catabolism such as glutaminolysis and the malateaspartate shuttle (Salway 2017). To account for all possible NADH redox pathways, we start with a detailed NADH redox model with $\mathrm{N}$ oxidases and $\mathrm{M}$ reductases (Figure 2a). NADH and NAD ${ }^{+}$ can bind and unbind to each oxidase and reductase. Once bound, NADH can be oxidized reversibly to $\mathrm{NAD}^{+}$by the oxidases, and $\mathrm{NAD}^{+}$can be reduced reversibly to NADH by the reductases, forming a redox cycle. A complete model of NADH redox reactions would include all of the other metabolites and enzymes associated with these reactions (Salway 2017). In the model described here (Figure 2a), the impact of these metabolites, enzymes, and other factors (i.e. pH, membrane potential, etc.) enter indirectly by affecting the NADH binding and unbinding rates and reactions rates with the oxidases and reductases (Figure 2-figure supplement 1).

FLIM does not resolve the association of NADH with individual enzymes, but quantifies the global bound and free state of NADH. Therefore to relate the FLIM measurements to activities of mitochondrial metabolic pathways, we coarse-grain the detailed model to account for the global activities of the oxidation and reduction pathways in mitochondria. We achieve this by performing an analytically exact coarse-graining of all $\mathrm{N}$ oxidases into a single effective oxidase and of all $\mathrm{M}$ reductases into a single effective reductase, while preserving the global binding and unbinding fluxes and the global reaction fluxes through the oxidases and reductases (Appendix 2, Figure 2b). The preservation of global fluxes explicitly relates the effective binding and unbinding rates and the reaction rates of the coarse-grained model to those of the detailed model (Appendix 2, Figure 2-figure supplement 1). Thus, the coarse-grained model describes the global NADH redox cycle, without the loss of generality, in the following manner: NADH can be bound to the effective oxidase, $N A D H \cdot o x$, or to the effective reductase, $N A D H \cdot r e$, or can be in a freely diffusing state, $N A D H_{f}$. Hence, the concentration of NADH bound to enzymes is, $\left[N A D H_{b}\right]=[N A D H \cdot o x]+$ $[N A D H \cdot r e]$, and the total concentration of NADH is, $[N A D H]=\left[N A D H_{b}\right]+\left[N A D H_{f}\right]$. The kinetics of the effective oxidase and reductase are represented by the forward, $r_{o x}^{+}$, and reverse, $r_{o x}^{-}$, oxidation rates, and the forward, $r_{r e}^{+}$, and reverse, $r_{r e}^{-}$, reduction rates. The model also includes eight binding and unbinding rates of NADH and $\mathrm{NAD}^{+}$to the oxidase and reductase (Figure $2 \mathrm{~b}$ ). The coarse-grained reaction rates and binding and unbinding rates can be arbitrary functions of metabolite concentrations, enzyme concentrations, and other factors (i.e. $\mathrm{pH}$, membrane potential, etc.). Hence this is a generic model of NADH redox reactions. 

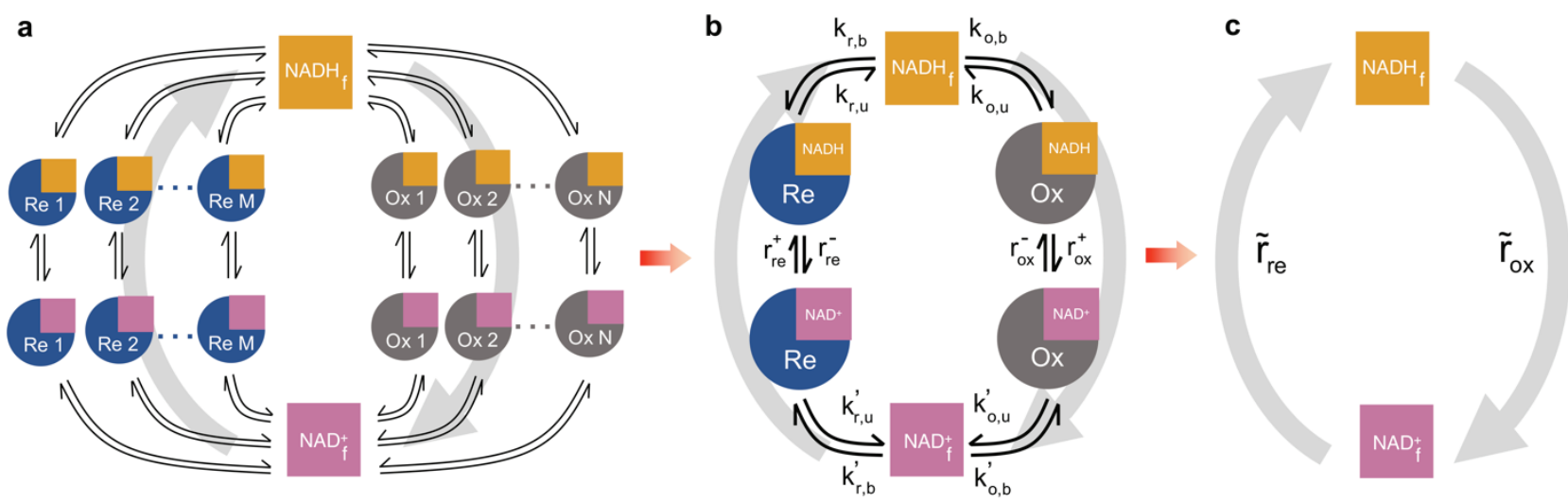

Figure 2 | Coarse-graining the NADH redox model. a, Schematic of the detailed NADH redox model. We consider all possible NADH redox pathways by modeling $\mathrm{N}$ oxidases $(\mathrm{Ox})$ and $\mathrm{M}$ reductases $(\mathrm{Re})$. Free $\mathrm{NADH}, N A D H_{f}$, and free $\mathrm{NAD}^{+}, N A D_{f}^{+}$, can bind and unbind with each oxidase and reductase. Once bound, NADH can be oxidized reversibly to $\mathrm{NAD}^{+}$by the oxidases, and $\mathrm{NAD}^{+}$can be reduced reversibly to NADH by the reductases, forming a redox cycle. b, Coarse-grained NADH redox model. All oxidases and reductases are coarse-grained into a single effective oxidase and reductase, respectively. $r_{o x}^{+}$and $r_{o x}^{-}$are the coarse-grained forward and reverse oxidation rates of the oxidase; $r_{r e}^{+}$and $r_{r e}^{-}$are the coarse-grained forward and reverse reduction rates of the reductase. $k_{o, b}, k_{o, u}, k_{r, b}, k_{r, u}$ and $k_{o, b}^{\prime}, k_{o, u}^{\prime}, k_{r, b}^{\prime}, k_{r, u}^{\prime}$ are the coarse-grained binding and unbinding rates of NADH and NAD ${ }^{+}$ to the oxidase and reductase. c. At steady state, all the kinetics of the model can be further coarse-grained into the turnover rate of free NADH, $\tilde{r}_{o x}$, and the turnover rate of free $\mathrm{NAD}^{+}, \tilde{r}_{r e}$, characterizing the two branches of the cycle.

\section{Accurately predicting mitochondrial metabolic flux from FLIM of NADH using the NADH redox model}

Our key theoretical result, is that this coarse-grained NADH redox model predicts that the oxidative flux of NADH is a product of the turnover rate and the concentration of free NADH (Figure 2c; Appendix 3):

$$
J_{o x} \equiv r_{o x}^{+}[N A D H \cdot o x]-r_{o x}^{-}\left[N A D^{+} \cdot o x\right]=\tilde{r}_{o x}\left[N A D H_{f}\right]
$$

where $\tilde{r}_{o x}=\alpha\left(\beta-\beta_{e q}\right)$. The turnover rate of free NADH, $\tilde{r}_{o x}$, is proportional to the difference between the NADH bound ratio, $\beta=f /(1-f)$, and the equilibrium NADH bound ratio, $\beta_{e q}$ (i.e. what the bound ratio would be if there was no net flux through the oxidase), with a constant of proportionality, $\alpha$, that is independent of the reaction rates of the oxidase and reductase. $\alpha$ and $\beta_{e q}$ can be explicitly related to the binding and unbinding rates of the model (Appendix 3, equation S29 and S31). If detailed biophysical models of the NADH oxidases are available, then parameters of these models can be explicitly mapped to the coarse-grained parameters of the NADH redox model. Appendix 4 and Table 1 contain mappings between the coarse-grained model and a number of previously proposed detailed biophysical models of NADH oxidation in the ETC (Beard, 2005; Korzeniewski and Zoladz, 2001; Hill, 1977; Jin and Bethke 2002; Chang et al., 2011). The central assumption required for the validity of Equation 1 is that the redox reactions, and binding and unbinding processes, can be approximated as being at steady-state (i.e. undergoing only quasistatic changes over perturbations or development).

In mitochondria, the major NADH oxidation pathway is the ETC. Thus, Equation 1 shows that there is a direct connection between quantities that can be measured by FLIM of NADH in 
mitochondria (i.e. $\beta$ and $\left[N A D H_{f}\right]$ ) and the flux through the ETC (i.e. $J_{o x}$ ). Equation 1 suggests a procedure for using FLIM to infer flux through the ETC: if a condition can be found under which there is no net flux through the ETC, then $\beta_{e q}$ can be measured with FLIM. Once $\beta_{e q}$ is known, then subsequent FLIM measurements of $\beta$ under other conditions allows $\tilde{r}_{o x}$, and hence $J_{o x}$, to be inferred (up to a constant of proportionality) (Appendix 5). We applied this procedure to analyze our oxygen drop experiments (Figure 1) by assuming that there was no net flux through the ETC at the lowest oxygen level achieved for each oocyte (implying that the measured value of $\beta$ at that oxygen concentration corresponds to $\beta_{e q}$ for that oocyte). This allowed us to obtain a prediction for $J_{o x}$ as a function of oxygen concentration for the oocytes (Figure 3a). To test these predictions, we directly determined $J_{o x}$ as a function of oxygen concentration by measuring the oxygen consumption rate (OCR) of the oocytes using a nanorespirometer (Lopes et al., 2005) (Methods). The direct measurements of $J_{o x}$ from OCR quantitatively agrees with the predictions of $J_{o x}$ from FLIM for all oxygen concentrations (Figure 3a), strongly arguing for the validity of the model and the inference procedure.

We next explored the impact of other perturbations. We first exposed oocytes to sodium oxamate, an inhibitor of lactate dehydrogenase (LDH), which resulted in large changes in the autoflourescence of NADH in mitochondria, including a $29 \% \pm 2 \%$ decrease in intensity and a $10 \%$ $\pm 3 \%$ increase in bound ratio (Figure $3 \mathrm{~b}$ ). We dropped oxygen levels to determine $\beta_{e q}$, and applied Equation 1 to infer the impact of sodium oxamate on $J_{o x}$ at $50 \mu \mathrm{M}$ oxygen (i.e. control levels of oxygen). Surprisingly, this procedure revealed that while the addition of sodium oxamate greatly impacts FLIM parameters, it is predicted to not affect $J_{o x}(\mathrm{p}=0.94)$, which was confirmed by direct measurements of oocytes' OCR (Figure $3 b ; p=0.90$ ). We next exposed oocytes to FCCP, an uncoupler of oxidative phosphorylation, and repeated the procedure to use FLIM measurements to infer $J_{o x}$. This resulted in a predicted $32 \% \pm 7 \%$ increase in $J_{o x}$ from the addition of FCCP, a result which is statistically indistinguishable from the $37 \% \pm 11 \%$ increase measured by OCR (Figure $3 \mathrm{c} ; \mathrm{p}=0.78$ ). The quantitative agreement between predictions from FLIM and direct measurements from OCR under a variety of conditions (i.e. varying oxygen tension, sodium oxamate, and FCCP), demonstrates that Equation 1 can be successfully used to infer flux through the ETC.

The work described above used the relation $\tilde{r}_{o x}=\alpha\left(\beta-\beta_{e q}\right)$ to predict the flux through the ETC. We next show that the model also predicts a relationship between $\tilde{r}_{o x}$ and the fluorescence lifetime of enzyme-bound NADH, $\tau_{l}$, in mitochondria. This provides a second means to use the model to infer $\tilde{r}_{o x}$, and hence $J_{o x}$, from FLIM of NADH. Specifically, we assume that NADH bound to the oxidase have a different average lifetime, $\tau_{o x}$, than NADH bound to the reductase, $\tau_{r e}$, which is reasonable because NADH bound to different enzymes do exhibit different fluorescence lifetimes (Sharick et al., 2018). This assumption implies that the experimentally measured long lifetime of $\mathrm{NADH}$ in mitochondria, $\tau_{l}$, is a weighted sum of these two lifetimes, $\tau_{l}=$ $\tau_{o x} \frac{[N A D H \cdot o x]}{[N A D H \cdot o x]+[N A D H \cdot r e]}+\tau_{r e} \frac{[N A D H \cdot r e]}{[N A D H \cdot o x]+[N A D H \cdot r e]}$. This, in turn, leads to a non-trivial prediction of the NADH redox model: that $\tau_{l}$ is linearly related to $1 / \beta$ (Appendix 5, equation (S47)). Such a linear relationship is indeed observed in individual oocytes subject to oxygen drops (Figure $3 \mathrm{~d}$ ). Experimentally measuring the slope and offset between $\tau_{l}$ and $1 / \beta$, which vary under different conditions (Figure 3d), provides a second means to infer $\tilde{r}_{o x}$ from FLIM measurements (Figure 3- 
figure supplement 2). We next used the lifetime $\left(\tau_{l}\right)$ method and the bound ratio $(\beta)$ method to separately infer $\tilde{r}_{o x}$, and hence $J_{o x}$, in oocytes subject to a wide variety of conditions (varying oxygen levels, varying pyruvate levels, with sodium oxamate, FCCP, rotenone, and oligomycin). The two methods quantitatively agree under all conditions (Figure $3 \mathrm{e}, \mathrm{p}=0.98$ ), which is a strong self-consistency check that further supports the use of the model to infer ETC flux from FLIM measurements of NADH (Figure 3-figure supplement 1).


Figure 3 | Coarse-grained NADH redox model enables accurate prediction of flux through the ETC from FLIM measurements of NADH. a, Predicted flux through the ETC, $J_{o x}$, from the FLIM of NADH ( $\mathrm{n}=68$ oocytes) agrees quantitatively with $J_{o x}$ from oxygen consumption rate (OCR) measurements $(\mathrm{N}=3$ measurements, $\mathrm{n}=15$ oocytes per measurement) for all oxygen concentrations. $J_{o x}$ is normalized by its value at $50 \mu \mathrm{M}$ oxygen. $\mathbf{b}-\mathbf{c}$, relative changes in NADH intensity, bound ratio, predicted $J_{o x}$ from FLIM and measured $J_{o x}$ from OCR with perturbations of oxamate $(n=20, N=2)$ and FCCP $(n=28, N=2)$. Relative changes are calculated as the change of the quantity as a result of the perturbation normalized by the value of the quantify before the perturbation. Predicted $J_{o x}$ agrees with measured $J_{o x}$ in both cases (NS: $\mathrm{p}>0.05$ from two-sided two-sample t-test. $\mathrm{p}=0.90$ (b) and $\mathrm{p}=0.78$ (c)). d, NADH long lifetime, $\tau_{l}$, is linearly related to the inverse of $\mathrm{NADH}$ bound ratio, $1 / \beta$, from the oxygen drop experiment of individual oocytes 
treated with oxamate and rotenone (results from 5 representative oocytes are shown for each condition). Each shade corresponds to results from an individual oocyte (symbols are experimental measurements and dashed lines are linear fits). e, NADH turnover rate $\tilde{r}_{o x}$ obtained from NADH long lifetime agrees quantitatively with that from NADH bound ratio across all perturbations $(\mathrm{p}=0.98) . \tilde{r}_{o x}$ is normalized by its value in the AKSOM condition without perturbations. Error bars represent standard error of the mean (s.e.m).
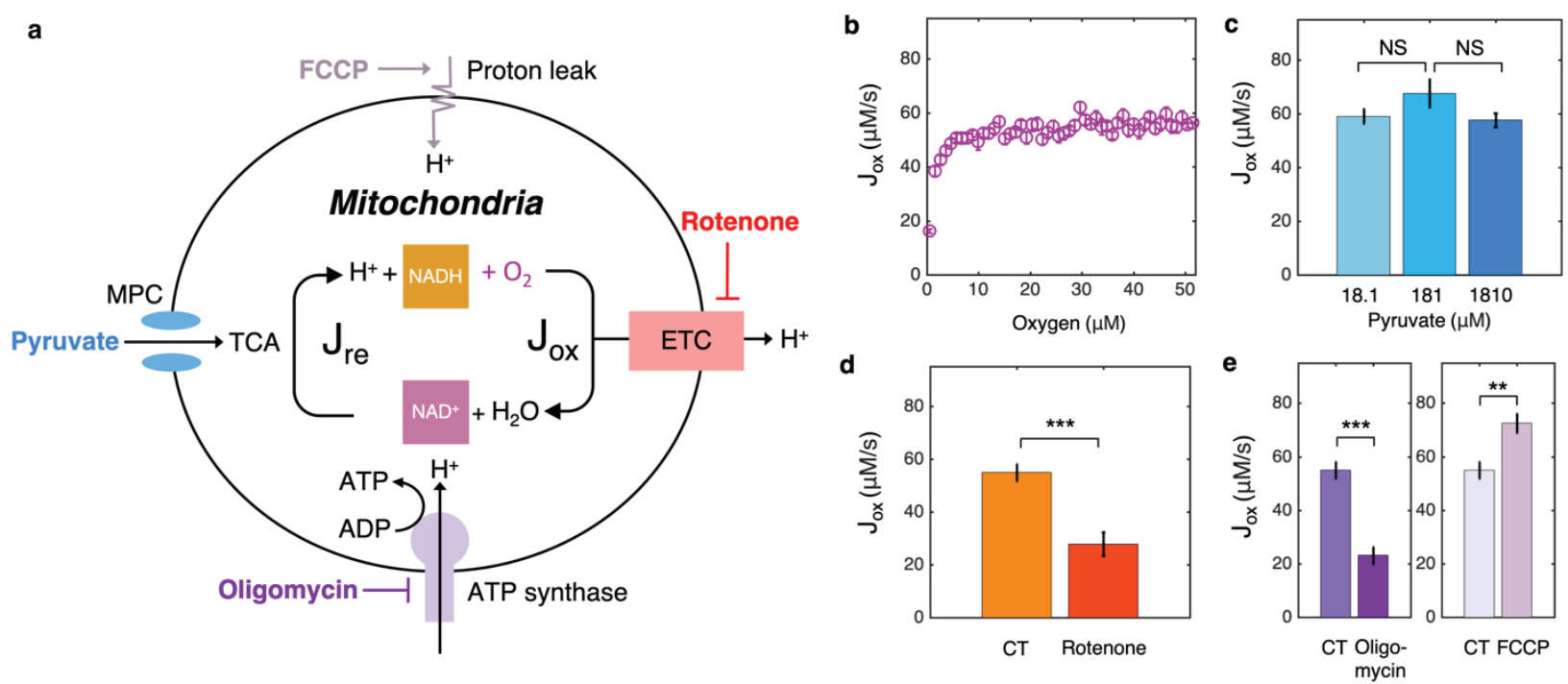

Figure 4 | Response of ETC flux to mitochondrial perturbations as inferred from FLIM of NADH. a, schematic of mitochondrial metabolism with biochemical perturbations. NADH redox cycle is composed of the oxidation pathway such as the ETC and the reduction pathway such as the TCA in mitochondria, where $\mathrm{J}_{\mathrm{ox}}$ and $\mathrm{J}_{\mathrm{re}}$ are the net metabolic fluxes through the oxidase and reductase for $\mathrm{NADH}$ and $\mathrm{NAD}^{+}$, respectively. At steady state, $J_{o x}=J_{r e}$. be, inferred ETC flux in response to oxygen $(n=68)$, pyruvate $(n=10,11,15$, from low to high concentrations), rotenone $(n=15)$, oligomycin $(n=13)$ and FCCP $(n=28)$ perturbations. CT is AKSOM with oxamate $(n=20)$. $n$ is the number of oocytes. Error bars represent standard error of the mean (s.e.m). NS: $p>0.05, * * *$ : $<<0.001, * *$ : $<<0.01$ from twosided two-sample t-test.

As described above, FLIM of NADH can be used to infer the turnover rate of free NADH because $\tilde{r}_{o x}=\alpha\left(\beta-\beta_{e q}\right)$, but the constant of proportionality cannot be determined by FLIM alone. Therefore, the redox model allows FLIM measurements to be used to quantitatively measure relative changes in flux through the ETC. If a separate measurement of flux through the ETC can be obtained at one condition, then the inferred relative changes from FLIM can be converted to absolute measurements. We used oocytes cultured in AKSOM media at $50 \pm 2 \mu \mathrm{M}$ oxygen as a reference state, which, from our OCR measurements yielded $J_{o x}=56.6 \pm 2.0 \mu \mathrm{M} \cdot \mathrm{s}^{-1}$ and hence a constant of proportionality between $\tilde{r}_{o x}$ and $\left(\beta-\beta_{e q}\right)$ of $\alpha=5.4 \pm 0.2 s^{-1}$ (Equation (S45)). Using this value of $\alpha$, we converted our measurements of relative changes in $\tilde{r}_{o x}$ under different conditions (Figure $3 \mathrm{e}$ ) to absolute values of $\tilde{r}_{o x}$ (Figure 4-figure supplement 1 ) and absolute values of $J_{o x}$ (Figure 4). Note that $J_{o x}$ is a flux density with units of concentration per second. Multiplying $J_{o x}$ by the volume of mitochondria in an oocyte gives the total ETC flux in that oocyte. The resulting inferred changes in $J_{o x}$ from FLIM are consistent with expectations (Figure $4 \mathrm{~b}-\mathrm{e}$ ). $J_{o x}$ is reduced upon decreasing oxygen level (oxygen is required to run the ETC), upon adding rotenone (which inhibits complex I of the ETC), and upon adding oligomycin (which inhibits ATP synthase and thereby hyperpolarizes mitochondria). $J_{o x}$ increases when FCCP is added (which depolarizes mitochondria). $J_{o x}$ is independent of pyruvate concentrations. 


\section{Revealing a subcellular spatial gradient of mitochondrial metabolic flux in mouse oocytes}

Our results presented so far comparing the predicted mitochondrial metabolic flux from FLIM with the directly measured mitochondrial metabolic flux from OCR were performed by averaging together FLIM measurements from all mitochondria within an oocyte (Figures 3 and 4). However, FLIM data is acquired with optical resolution, enabling detailed subcellular measurements. To see if there are spatial variations in FLIM measurements within individual oocytes, we computed the mean decay time of NADH for each mitochondrial pixel. The NADH decay times display a clear spatial gradient, with higher values closer to the oocyte center (Figure 5a).

To quantify this gradient in more detail, we partitioned mouse oocytes with equal-spaced concentric rings (Figure 5b), and fitted photon decay curves from mitochondrial pixels within each ring to obtain FLIM parameters as a function of distance from the oocyte center. NADH intensity, bound ratio and long lifetime in mitochondria all display significant spatial gradient within oocytes (Figure 5c). To further explore spatial variations in mitochondria in mouse oocytes, we next used the membrane potential-sensitive dye TMRM, which preferentially accumulates in mitochondria with higher membrane potential (AL-Zubaidi et al., 2019). We observed a strong spatial gradient of the intensity of TMRM in mitochondria within oocytes, with dimmer mitochondria near the cell periphery (Figure 5d, e), indicating that mitochondria near the periphery of the oocyte have a lower membrane potential. This result is robust to locally normalizing TMRM intensity by mitochondrial mass using a membrane insensitive dye (Mitotracker Red FM), or using an alternative membrane potential-sensitive dye, JC-1 (Figure 5-figure supplement 1).

Next, using Equation 1 and $\beta_{e q}$ obtained at the lowest oxygen level, and confirming that $\beta_{e q}$ is uniform within the oocyte with complete inhibition of ETC using rotenone (Figure 5-figure supplement 2), we predicted the ETC flux, $J_{o x}$, as a function of distance from the oocyte's center. The mitochondrial flux displayed a strong spatial gradient within oocytes, with a higher flux closer to the cell periphery (Figure 5f). Plotting the predicted mitochondrial metabolic flux vs TMRM intensity shows a strong negative correlation between these two variables (Figure $5 \mathrm{~g}$ ), indicating that mitochondrial metabolic flux increases with decreasing mitochondrial membrane potential throughout oocytes. Interestingly, a similar trend is observed upon inhibiting ATP-synthase, which decreases flux (Figure 4e, oligomycin) and increases membrane potential (Brand and Nicholls, 2011), or enhancing proton leak, which increases flux (Figure 4e, FCCP) and decreases membrane potential (Brand and Nicholls, 2011). Thus, the subcellular spatial variation in mitochondrial metabolic flux that we discovered (Figure 5f) might be caused by spatially varying ATP-synthesis or proton leak in mitochondria in mouse oocytes. 
a

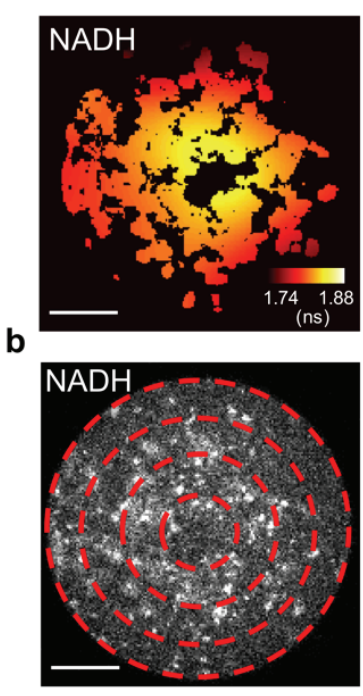

c

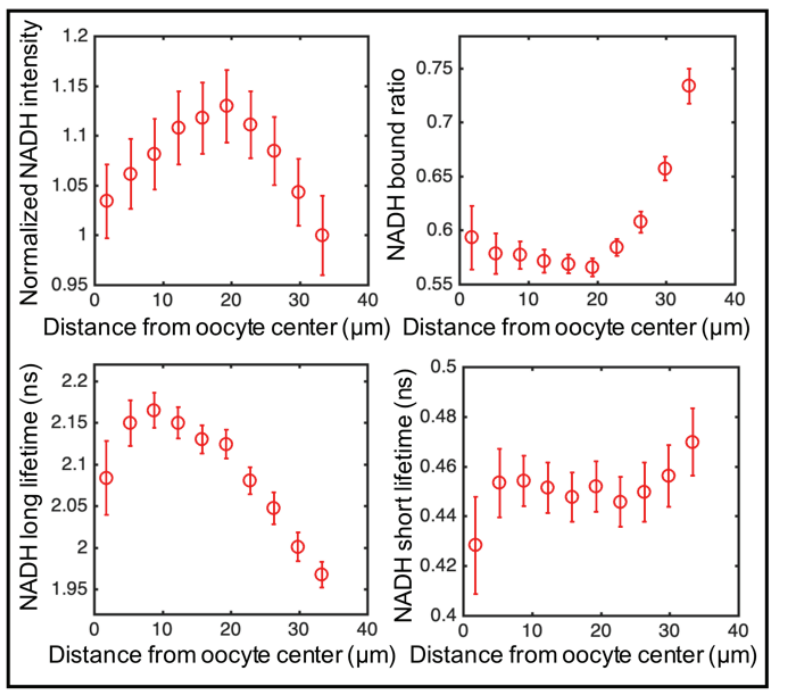

d

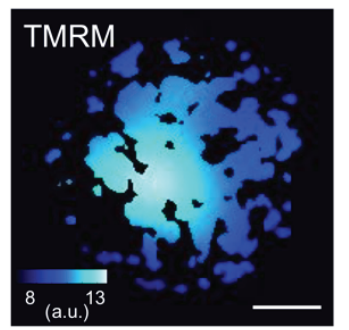

e

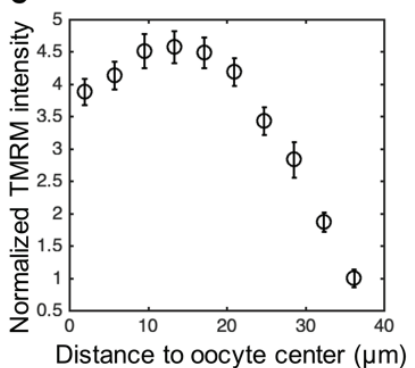

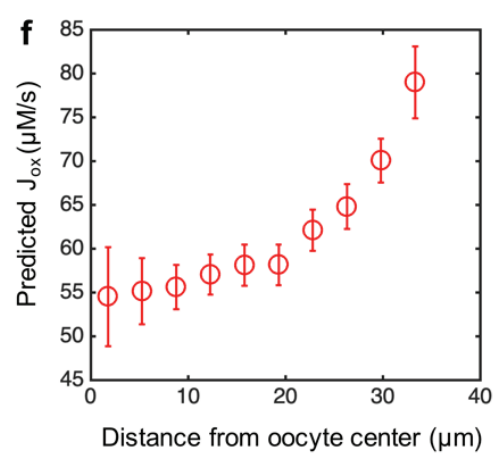

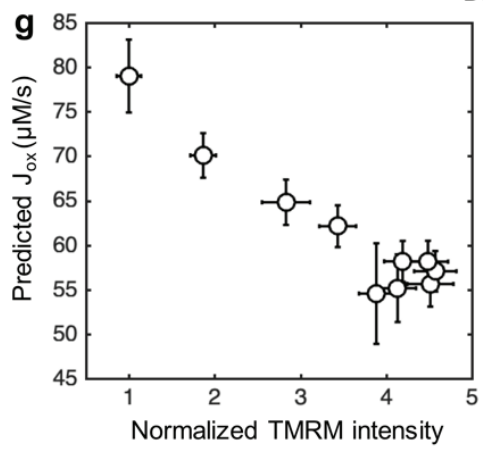

Figure 5 | Subcellular spatial gradient of mitochondrial metabolism in mouse oocytes. a, Heatmap of the mean NADH decay time in mitochondria exhibits a subcellular spatial gradient within oocytes. b, NADH intensity image of the oocyte partitioned with equal-spaced concentric rings. c, Mitochondrial normalized NADH intensity (upper left), bound fraction $f$ (upper right), long fluorescence lifetime $\tau_{l}$ (lower left), and short fluorescence lifetime $\tau_{s}$ (lower right) as a function of distance from the oocyte center $(\mathrm{n}=67)$. $\mathbf{d}$, Heatmap of the TMRM intensity in mitochondria, which increases with mitochondrial membrane potential, exhibits a subcellular spatial gradient within oocytes $(n=16)$. e, Normalized TMRM intensity as a function of distance from the oocyte center. f, Predicted mitochondrial ETC flux from FLIM of NADH as a function of distance from the oocyte center $(n=67)$. $g$, Subcellular variation in mitochondrial flux correlates with a corresponding subcellular variation in mitochondrial membrane potential. Scale bar $20 \mu \mathrm{m}$. Error bars represent standard error of the mean (s.e.m).

\section{Discussion}

\section{The NADH redox model is a general model to relate FLIM measurements of NADH to mitochondrial metabolic fluxes}

Despite extensive studies and applications of FLIM in metabolic research (Bird et al., 2005; Skala et al., 2007; Heikal, 2010; Sharick et al., 2018; Sanchez et al., 2018; Liu et al., 2018; Sanchez et al., 2019; Ma et al., 2019), it remains a challenge to relate FLIM measurements to the activities of the underlying metabolic pathways in cells. We overcome this challenge by developing a coarsegrained NADH redox model to relate FLIM measurements to mitochondrial metabolic fluxes. The model is constructed by explicitly coarse-graining a detailed NADH redox model with an arbitrary 
number of oxidases and reductases that represent all the possible enzymes involved in NADH redox reactions. The coarse-graining reduces all oxidases to an effective oxidase and all reductases to an effective reductase. The kinetic rates of the coarse-grained model are related to those of the detailed model by preserving the global fluxes through the oxidases and the reductases. In mitochondria, the coarse-grained oxidase represents the ETC. We have shown that the coarsegrained model predicts that the flux through the ETC is a product of the turnover rate and the concentration of free NADH (Equation 1). The turnover rate is proportional to the difference between the nonequilibrium and the equilibrium NADH bound ratio measured by FLIM of NADH. This model hence provides a generic framework to relate FLIM measurements of NADH to the flux through the ETC in mitochondria.

The model prediction of the ETC flux in Equation (1) is valid even if all of the reaction rates, and binding and unbinding rates, are arbitrary functions of metabolite concentrations, enzyme concentrations, and other variables (such as membrane potential, oxygen concentration, and the presence of added drugs). Remarkably, all the kinetic rates enter Equation (1) through only two effective parameters: $\alpha$ and $\beta_{e q}$. We determined the value of $\alpha$ from an OCR measurement (Appendix 5, equation (S45)), and we determined the value of $\beta_{e q}$ from FLIM of NADH at low oxygen levels (Appendix 5, Figure 3-figure supplement 1). In MII mouse oocyte, $\alpha$ does not significantly vary in response to drug and nutrient perturbations. This is demonstrated by the agreement between predicted ETC flux and measured OCR with a constant $\alpha$ of $5.4 \pm 0.2 s^{-1}$. In contrast, $\beta_{e q}$ does vary with these perturbations (Figure 3-figure supplement 1). Using these two parameters, we inferred the turnover rate of free NADH, $\tilde{r}_{o x}$, from FLIM measurements of NADH (Figure 4-figure supplement 1). By multiplying this turnover rate with the concentration of free $\mathrm{NADH},\left[\mathrm{NADH}_{f}\right]$, we obtained the ETC flux from Equation (1) (Figure 4). Thus, all the complex behaviors of the binding and unbinding and reaction rates are captured by the variations in FLIM parameters of $\mathrm{NADH}$, and our coarse-grained model provides a generic way to interpret these variations.

Finally, for mouse oocyte, $\beta_{e q}$ is smaller than $\beta$, and $\alpha$ has a positive value. This does not have to be the case, since the model also admits solutions with $\beta_{e q}$ larger than $\beta$ and a negative $\alpha$ (Appendix 3, equation (S29)). Hence if a perturbation is observed to decrease the NADH bound ratio $\beta$, it does not necessarily imply a decrease of the ETC flux. A similar argument holds for the long lifetime of NADH, where a decrease of NADH long lifetime is not always associated with an increase of the ETC flux (Appendix 5, equation (S47)). Rather, measurements of $\alpha$ and $\beta_{e q}$ are required to use Equation 1 to infer ETC flux from FLIM of NADH.

\section{The underlying assumptions and limitations of the NADH redox model}

In this section, we clarify the underlying assumptions and limitations of the model to facilitate accurate interpretation of FLIM of NADH in different biological contexts.

To use the coarse-grained NADH model, segmentation needs to be performed to separate mitochondrial NADH signal from cytoplasmic NADH signal, because they encode different metabolic fluxes. In mouse oocyte, the segmentation can be reliably performed based on NADH 
images due to the higher NADH intensity in mitochondria than cytoplasm. Mitochondrial movements are also slow in MII oocyte (Video 1), hence longer exposure times can be used to obtain high contrast NADH images. For cells where NADH contrast is low, such as in yeast cells (Papagiannakis et al., 2016; Shaw and Nunnari, 2002), mitotracker dye (Appendix 1, Figure 1figure supplement 1) or mitochondrial associated fluorescent proteins (Westermann and Neupert, 2000) will likely be needed for reliable segmentation of mitochondria.

One of the most important assumptions that enables the coarse-grained model to be used to predict fluxes is that the NADH redox cycle can be well approximated as being at steady state, i.e. the rate of change of NADH concentrations is much slower than the kinetic rates, including the binding/unbinding rates and the reaction rates. This is true for mouse oocyte, where the NADH intensity does not significantly change over the course of hours. This assumption also holds for slow processes such as cell cycles (Papagiannakis et al., 2016), which occurs on the timescale of hours compared to timescales of seconds for the kinetic rates. The steady-state approximation could fail for rapid dynamics of NADH, such as the transient overshoot of NADH in neurons induced by acute external stimulus (Díaz-García et al., 2020).

Another assumption is that the dominant autofluorescence FLIM signal comes from NADH, while $\mathrm{NADPH}$, which shares a similar spectrum as NADH, is negligible in mitochondria. This assumption is valid for mouse oocyte, where NADH concentration is 40 times greater than the concentration of NADPH (Bustamante et al., 2017). NADPH autofluorescence might be significant for other cells such as yeast (Park et al., 2016).

Finally, when relating NADH FLIM measurements to the ETC flux we did not explicitly consider the contribution to the flux through $\mathrm{FADH}_{2}$. This is a valid approximation when the $\mathrm{FADH}_{2}$ oxidative flux is much smaller than the NADH oxidative flux, as is often the case since pyruvate dehydrogenase plus the TCA cycle yields 4 NADH molecules but only one $\mathrm{FADH}_{2}$ molecule per cycle. Alternatively, if the $\mathrm{FADH}_{2}$ flux is proportional to the NADH flux then a rescaled value of $\alpha$ can be used in Equation 1 to effectively account for both fluxes. While a proportionality of $\mathrm{FADH}_{2}$ flux and NADH flux is expected under some circumstances, it will not generally hold since $\mathrm{FADH}_{2}$ and NADH can be independently reduced in mitochondria, for example, via the glycerol phosphate shuttle.

Given these underlying assumptions, the model needs to be tested before being applied to different biological systems. The present study provides an example for such tests in mouse oocytes by comparing the predicted ETC flux with directly measured oxygen consumption rate across a wide range of perturbations (Figure 3).

\section{Towards spatiotemporal regulations of metabolic fluxes in cells and tissues}

By employing the coarse-grained model described above, FLIM of NADH can be used to measure spatial variations in metabolic fluxes. We used this approach to discover subcellular spatial gradient of mitochondrial metabolic fluxes in mouse oocytes. Metabolic heterogeneities, between and within individual cells, are believed to be widespread, but remain poorly characterized (Takhaveev and Heinemann 2018; Aryaman et al., 2019). We hope that this combination of coarse- 
grained modeling and FLIM of NADH will also prove useful in studying metabolic heterogeneities in other systems.

\section{Materials and methods}

\section{Culturing of mouse oocytes}

Frozen MII mouse oocytes (Strain B6C3F1) were purchased from EmbryoTech. Oocytes were thawed and cultured in droplets of AKSOM media purchased from Millipore Sigma in plastic petri dish. Mineral oil from VitroLife was applied to cover the droplets to prevent evaporation of the media. Oocytes were then equilibrated in an incubator at $37^{\circ} \mathrm{C}$, with $5 \% \mathrm{CO}_{2}$ and air saturated oxygen before imaging. For imaging, oocytes were transferred to a $2 \mu 1$ media droplet in a $35 \mathrm{~mm}$ glass bottom FluoroDish from WPI covered with $400-500 \mu l$ of oil. The glass bottom dish was placed in an ibidi chamber with temperature and gas control during imaging. Temperature was maintained at $37^{\circ} \mathrm{C}$ via heated chamber and objective heater. $\mathrm{CO}_{2}$ was maintained at $5 \%$ using gas tanks.

\section{FLIM measurements}

Our FLIM system consists of a two-photon confocal microscope with a 40X 1.25NA water immersion Nikon objective, Becker and Hickle Time Correlated Single Photon Counting (TCSPC) acquisition system and a pulsed MaiTai DeepSee Ti:Sapphire laser from Spectra-Physics. NADH autofluorescence was obtained at 750nm excitation wavelength with a 460/50nm emission filter. Laser power at the objective was maintained at $3 \mathrm{~mW}$. The scanning area was 512 by 512 pixels with a pixel size of $420 \mathrm{~nm}$. Acquisition time was 30 seconds per frame. A histogram of NADH decay times was obtained at each pixel of the image.

\section{Oxygen measurement}

Oxygen level was measured in the Ibidi chamber with an electrode-based oxygen sensor (Gaslab). Since the oil layer covering the media droplet was very thin, the oxygen level in the droplet was assumed to be in instant equilibration with the chamber.

\section{Image and FLIM data analysis}

To separate mitochondrial NADH signal from cytoplasmic signal, we performed machine learning based segmentation algorithms on NADH intensity images. We used the freeware ilastik (Berg et al., 2019), which implements a supervised learning algorithm for pixel classification. The classifiers were trained to separate mitochondrial pixels from cytoplasmic pixels with a greater than $80 \%$ accuracy, as tested by MitoTracker Red FM (Appendix 1, Figure 1-figure supplement 1). We grouped photons from all mitochondrial pixels to obtain a histogram of NADH decay times for each oocyte. To extract the FLIM parameters of NADH bound fraction $f$, long lifetime $\tau_{l}$ and short lifetime $\tau_{s}$, we fitted the histogram with $G=I R F *\left(C_{1} F+C_{2}\right)$, where $*$ indicates a convolution, and IRF is the instrument response function of the FLIM system, measured using a 
urea crystal. $F(\tau)=f \cdot \exp \left(-\frac{\tau}{\tau_{l}}\right)+(1-f) \cdot \exp \left(-\frac{\tau}{\tau_{s}}\right)$ is the two-exponential model for the NADH fluorescence decay. $C_{1}$ is the amplitude of the decay and $C_{2}$ is the background noise. The fitting was performed with an custom MATLAB code using a Levenberg-Marquardt algorithm. To obtain the intensity, $I$, of mitochondrial NADH, we first measured the average number of photons per mitochondrial pixel, and divided it by the pixel area, $0.185 \mu \mathrm{m}^{2}$, and pixel scanning time $4.09 \mu \mathrm{s}$. The flux of ETC is inferred using equation 1 for each oocyte. Heatmaps of mean NADH decay times were obtained by computing NADH decay time of each mitochondrial pixel and averaging over neighboring mitochondrial pixels weighted by a gaussian kernel with a standard deviation of 20 pixels. All FLIM measurements were taken from distinct individual oocytes and error bars in all figures of FLIM represent standard error of the mean across different individual oocytes. Number of oocytes is reported with $n$.

\section{Metabolic perturbations}

Oxygen drop experiments were performed by mixing nitrogen-balanced 5\% $\mathrm{O}_{2}$ gas with $0 \% \mathrm{O}_{2}$ gas at different ratios to create a continuous oxygen drop profile. $\mathrm{CO}_{2}$ was maintained at 5\%. Oocytes were imaged for $10 \mathrm{mins}$ at $5 \% \mathrm{O}_{2}, 30 \mathrm{mins}$ during the continuous drop from $5 \% \mathrm{O}_{2}$ to approximately $0 \% \mathrm{O}_{2}$, and 20 mins after quickly returning to $5 \% \mathrm{O}_{2}$. Oxygen levels were simultaneously monitored with an electrode-based oxygen sensor in the ibidi chamber. 5\% $\mathrm{O}_{2}$ corresponds to $\sim 50 \mu \mathrm{M}$ of oxygen concentration in the culturing media. All the drug perturbations were performed by equilibrating oocytes in the AKSOM media containing the corresponding drug for 15-30mins before the oxygen drop experiments. $9 \mathrm{mM}$ sodium oxamate, $15-60 \mu \mathrm{M}$ rotenone, 5$50 \mu \mathrm{M}$ oligomycin and 1-50 $\mu \mathrm{M}$ FCCP were used for the perturbations. Pyruvate perturbations were performed by making KSOM media following Cold Spring Harbor Laboratory protocols with varying concentrations of sodium pyruvate. For oligomycin, FCCP, rotenone and pyruvate perturbations, $9 \mathrm{mM}$ of sodium oxamate was also added to the media to suppress cytoplasmic NADH signal for better mitochondrial segmentation. The addition of the oxamate does not change the ETC flux of the mitochondria. All drugs were purchased from Sigma Aldrich. Temperature was maintained at $37^{\circ} \mathrm{C}$. Oocytes were selected and assigned to different perturbation groups randomly. Data were also collected in random order for different oocytes.

\section{Oxygen consumption rate (OCR) measurement}

The oxygen consumption rate of the oocytes was measured using the nanorespirometer from Unisense (Lopes et al., 2005). A batch of 15 oocytes were placed at the bottom of a glass capillary with a diameter of $0.68 \mathrm{~mm}$ and a height of $3 \mathrm{~mm}$. The capillary well is filled with AKSOM media. After an equilibration time of $\sim 2$ hours, a steady state linear oxygen gradient is established in the capillary well due to the balance of oocyte respiration and oxygen diffusion. An electrode-based oxygen sensor (Unisense) is used to measure the oxygen gradient. The oxygen consumption rate is calculated as the product of the oxygen gradient, diffusivity of oxygen in the media, taken to be $3.37 \times 10^{-5} \mathrm{~cm}^{2} / \mathrm{s}$, and the cross sectional area of the capillary well, which was $0.36 \mathrm{~mm}^{2}$. The entire system was enclosed in a custom built chamber with temperature and gas control. Temperature was maintained at $37^{\circ} \mathrm{C}$. Oxygen level was continuously varied during oxygen drop experiments, and maintained at the air saturation level for drug and pyruvate perturbations. All OCR measurements were taken from distinct batches of oocytes in groups of 15. Error bars in all 
figures of OCR represent standard error of the mean across different groups of oocytes normalized by the number of oocytes in each group. Number of oocytes is reported with $n$. Number of batches is reported with $\mathrm{N}$.

\section{Mitochondrial membrane potential measurement}

The spatial distribution of mitochondrial membrane potential within oocytes was measured with a potential-sensitive dye TMRM (Sigma Aldrich). Oocytes were cultured in AKSOM with 100nM TMRM for 30 minutes before imaging. Heatmaps of TMRM intensity were obtained by computing photon counts for each mitochondrial pixel and averaging over neighboring mitochondrial pixels weighted by a gaussian kernel with a standard deviation of 20 pixels. To normalize TMRM intensity by mitochondrial mass, we cultured oocytes in AKSOM with 100nM Mitotracker Red FM and 25nM TMRM for 30 minutes before imaging. We also cultured oocytes in AKSOM with $1 \mu \mathrm{g} / \mathrm{ml} \mathrm{JC}-1$ dye for 3 hours before imaging. 


\section{Appendix 1}

\section{Segmentation of mitochondria and calculation of NADH concentrations}

\section{Segmentation of mitochondria}

We used Ilastik, a machine-learning-based software for image analysis, to classify pixels in the NADH images containing mitochondria (Berg et al., 2019). For each experiment, we generated a time lapse movie of NADH (Video 1). We used a few images in the movie as the training data set to train the software to classify mitochondrial pixels by manually selecting clustered high brightness pixels. Other pixels are classified as either cytoplasm or background. We then applied the trained pixel classifier to generate a mitochondrial probability map for each image in the entire movie, with each pixel assigned a probability between 0 to 1 to be mitochondrial pixel. Pixels with a probability higher than 0.7 were considered to be mitochondrial pixels.

To test the accuracy of this segmentation algorithm, we immersed the oocytes in media containing MitoTracker Red FM, a dye that specifically labels mitochondria. Pixels with intensity above 60 percentile in the MitoTracker image were considered to be mitochondrial pixels. We imaged $\mathrm{NADH}$ and MitoTracker for the same oocyte and compared the resulting distribution of mitochondria (Figure 1-figure supplement 1). The accuracy of the NADH-based segmentation of mitochondria is $80.6 \pm 1.0 \%(\mathrm{SEM})$ as averaged over 7 oocytes.

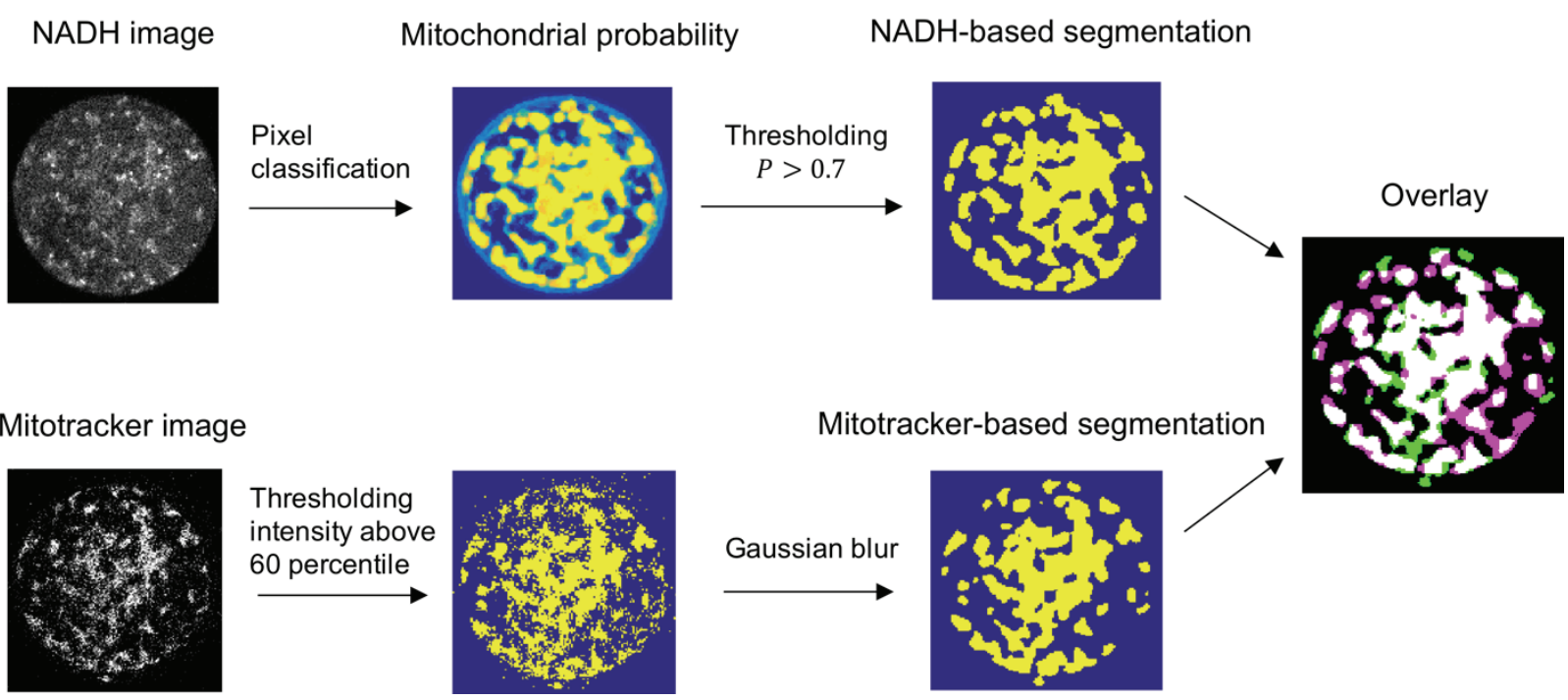

Figure 1-figure supplement 1 | Machine learning based segmentation of mitochondria from NADH images. The NADH-based segmentation image is overlaid with MitoTracker-based segmentation image. The white region corresponds to the overlap. The accuracy of the NADH-based segmentation is quantified as the ratio of the photon count from the overlap pixels to the photon count from mitochondrial pixels based on NADH segmentation. 


\section{Converting NADH intensity to NADH concentrations}
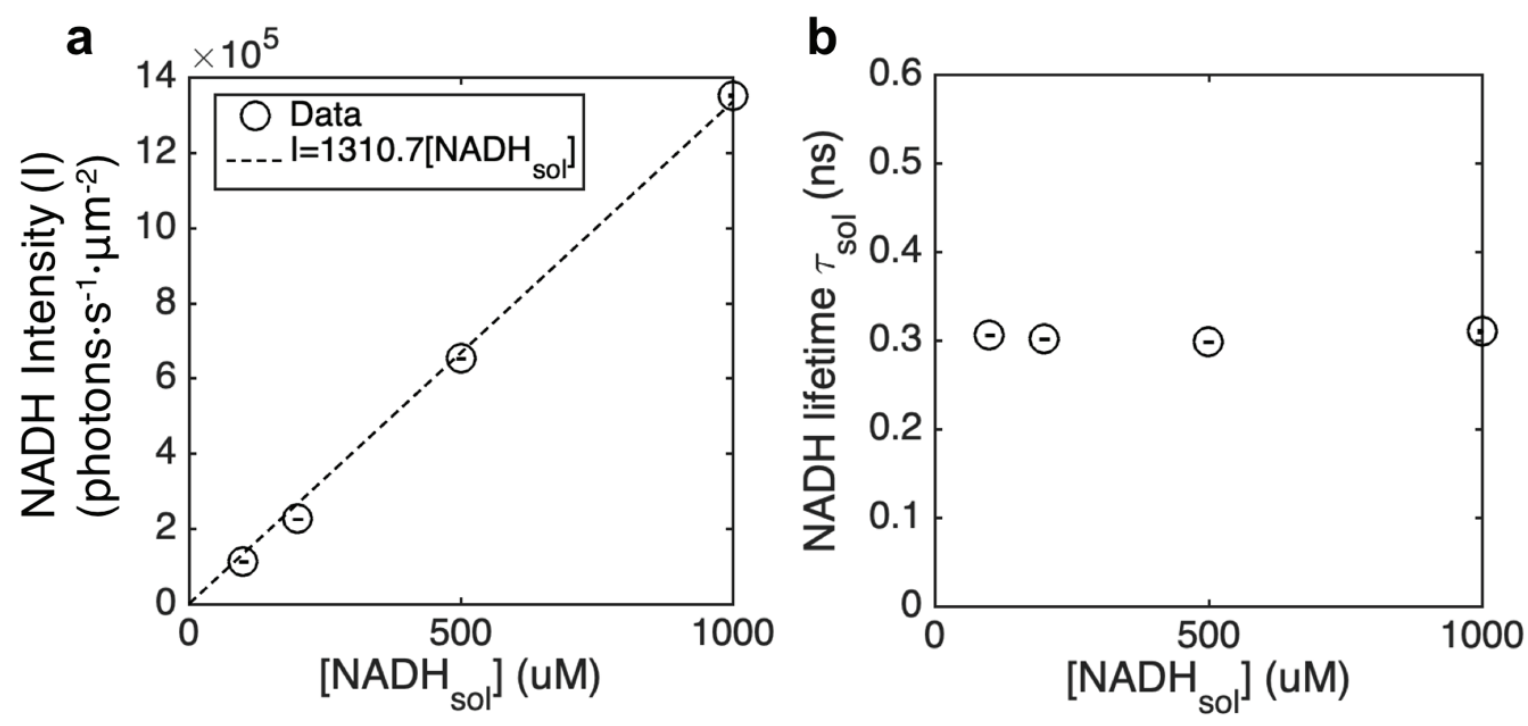

Figure 1-figure supplement 2 | Calibration and conversion of NADH concentrations from fluorescence intensities in vitro. a, NADH intensity vs titrated NADH concentrations in AKSOM solution. b, fluorescence lifetime of $\mathrm{NADH}$ in AKSOM solution.

Since the molecular brightness of NADH depends on the fluorescence lifetime of NADH, which changes drastically upon binding enzymes, the NADH concentration is not linearly proportional to NADH intensity. FLIM provides an accurate way of measuring NADH concentrations by simultaneously measuring fluorescence intensity and lifetime. We now derive the NADH intensity-concentration relation from the FLIM measurements. Assuming molecular brightness is proportional to the fluorescence lifetime, and therefore that free and bound NADH have different contributions to the measured intensity, we have

$$
I=c_{s} \tau_{s}\left[N A D H_{f}\right]+c_{s} \tau_{l}\left[N A D H_{b}\right](S 1)
$$

where $I$ is the intensity of NADH and $c_{s}$ is a calibration factor that depends on the laser power. From equation (S1), we obtained the concentrations of free and bound NADH:

$$
\begin{aligned}
{\left[N A D H_{f}\right] } & =\frac{I(1-f)}{c_{s}\left[\left(\tau_{l}-\tau_{s}\right) f+\tau_{s}\right]}(S 2), \\
{\left[N A D H_{b}\right] } & =\left[N A D H_{f}\right] \frac{f}{1-f}(S 3),
\end{aligned}
$$

where $f$ is the fraction of bound NADH.

To get the calibration factor $c_{S}$, we titrated NADH in AKSOM solutions and fitted the calibration curve using:

$$
I=c_{s} \tau_{\text {sol }}\left[N A D H_{s o l}\right](S 4),
$$


where $\tau_{\text {sol }}$ is the lifetime of NADH in solution. $\tau_{\text {sol }}$ was directly measured by FLIM (Figure 1figure supplement $2 \mathrm{~b}$ ), allowing us to obtain $c_{S}$ from the fit (Figure 1-figure supplement 2a). Using equations (S2)-(S3), we obtained the free and bound NADH concentrations in mouse oocytes for oxygen drop (Figure 1c) and drug perturbations (Figure 3-figure supplement 1e-f).

\section{FLIM can be used to accurately measure concentrations of bound and free NADH in vitro}
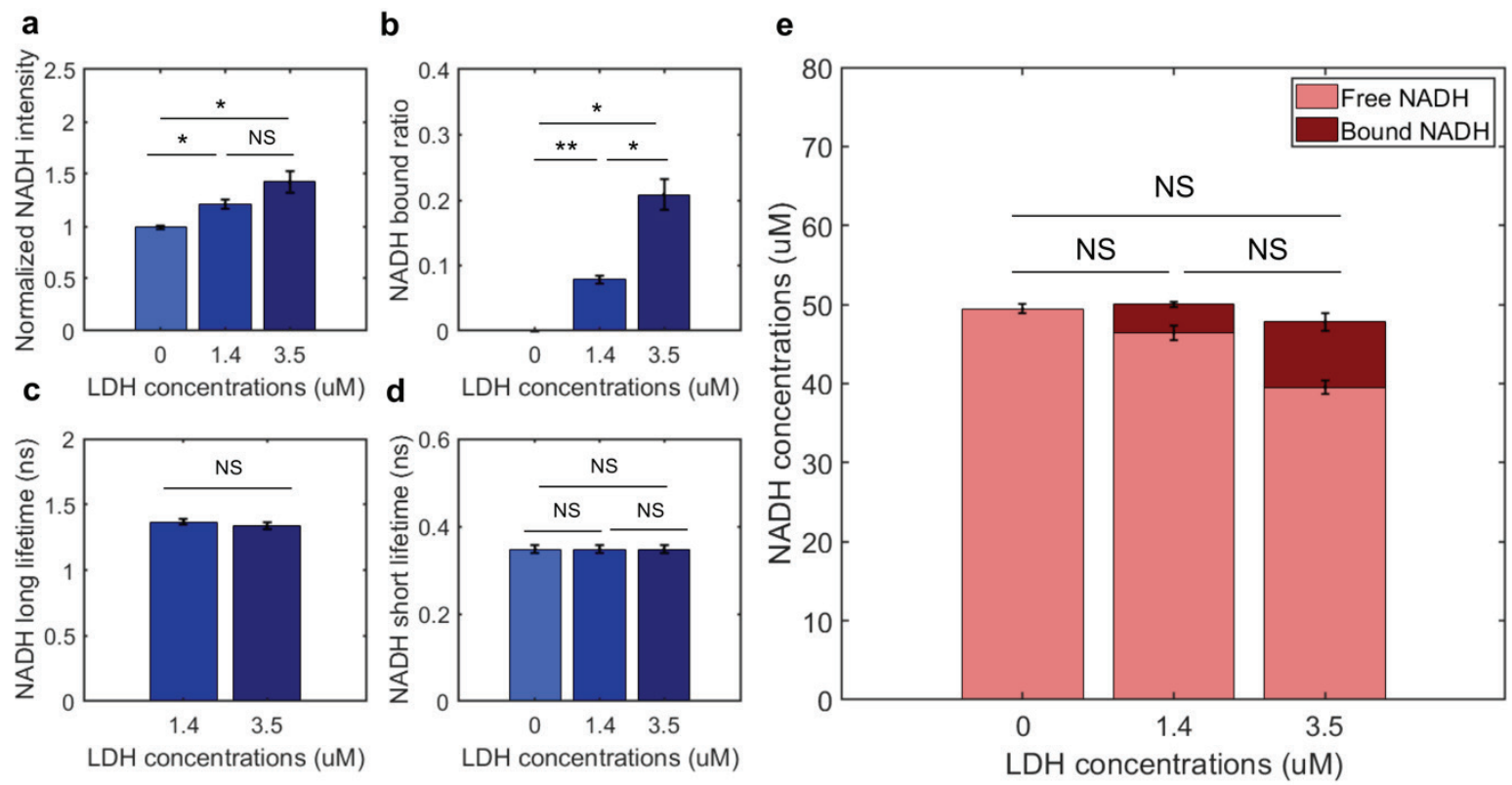

Figure 1-figure supplement 3 | Measurement of concentrations of free and bound NADH in vitro from FLIM of NADH. a-d, NADH intensity, bound ratio, long lifetime and short lifetime from FLIM of NADH with various concentrations of lactate dehydrogenase (LDH) in vitro. e. Concentrations of free and bound NADH calculated from FLIM of NADH. Error bars are standard error across replicates. $\mathrm{N}=2$. Student's t-test is performed. ${ }^{*} \mathrm{p}<0.05,{ }^{* *} \mathrm{p}<0.01$, $* * * \mathrm{p}<0.001$.

To test if absolute concentrations of free and bound NADH can be accurately measured from FLIM of NADH, we prepared solutions with known total concentration of NADH, and titrated the concentration of lactate dehydrogenase (LDH), an enzyme to which NADH can bind. We prepared the solutions with $50 \mathrm{mM}$ TRIS buffer, $150 \mathrm{mM} \mathrm{NaCl}$ at $\mathrm{pH} 7.6$ and $37^{\circ} \mathrm{C}$. We added a total concentration of $50 \mu \mathrm{M} N A D H$ to the solution and titrated $\mathrm{LDH}$ concentrations at $0,1.4 \mu \mathrm{M}$ and $3.5 \mu \mathrm{M}$. We first performed single exponential fitting of the NADH decay curve at $0 \mu \mathrm{M} \mathrm{LDH}$, where all NADH are free, to obtain the NADH short lifetime (Figure 1-figure supplement 3d). From the NADH intensity (Figure 1-figure supplement 3a), we obtained the calibration factor $c_{s}$ using equation (S4) with $\left[N A D H_{\text {sol }}\right]=50 \mu M$. We then fixed the short lifetime and performed two-exponential fitting of the NADH decay curve at $\mathrm{LDH}$ concentrations of $1.4 \mu \mathrm{M}$ and $3.5 \mu \mathrm{M}$ to obtain the bound ratio (Figure 1-figure supplement 3b) and long lifetime (Figure 1-figure supplement 3c). As expected, NADH bound ratio increases with LDH concentrations, as there is more enzyme for NADH to bind. Finally, we calculated free NADH concentration $\left[N A D H_{f}\right]$ and bound NADH concentration $\left[N A D H_{b}\right]$ using equations (S2) and (S3) from the FLIM parameters. Remarkably, the free and bound concentrations of NADH both change with LDH concentrations but the total concentration remains at $50 \mu \mathrm{M}$ (Figure 1-figure supplement 3e). This result shows 
that equations (S2)-(S3) can be used to accurately measure the concentrations of free and bound NADH from FLIM measurements of NADH.

\section{Appendix 2}

\section{Coarse-graining the detailed NADH redox model}
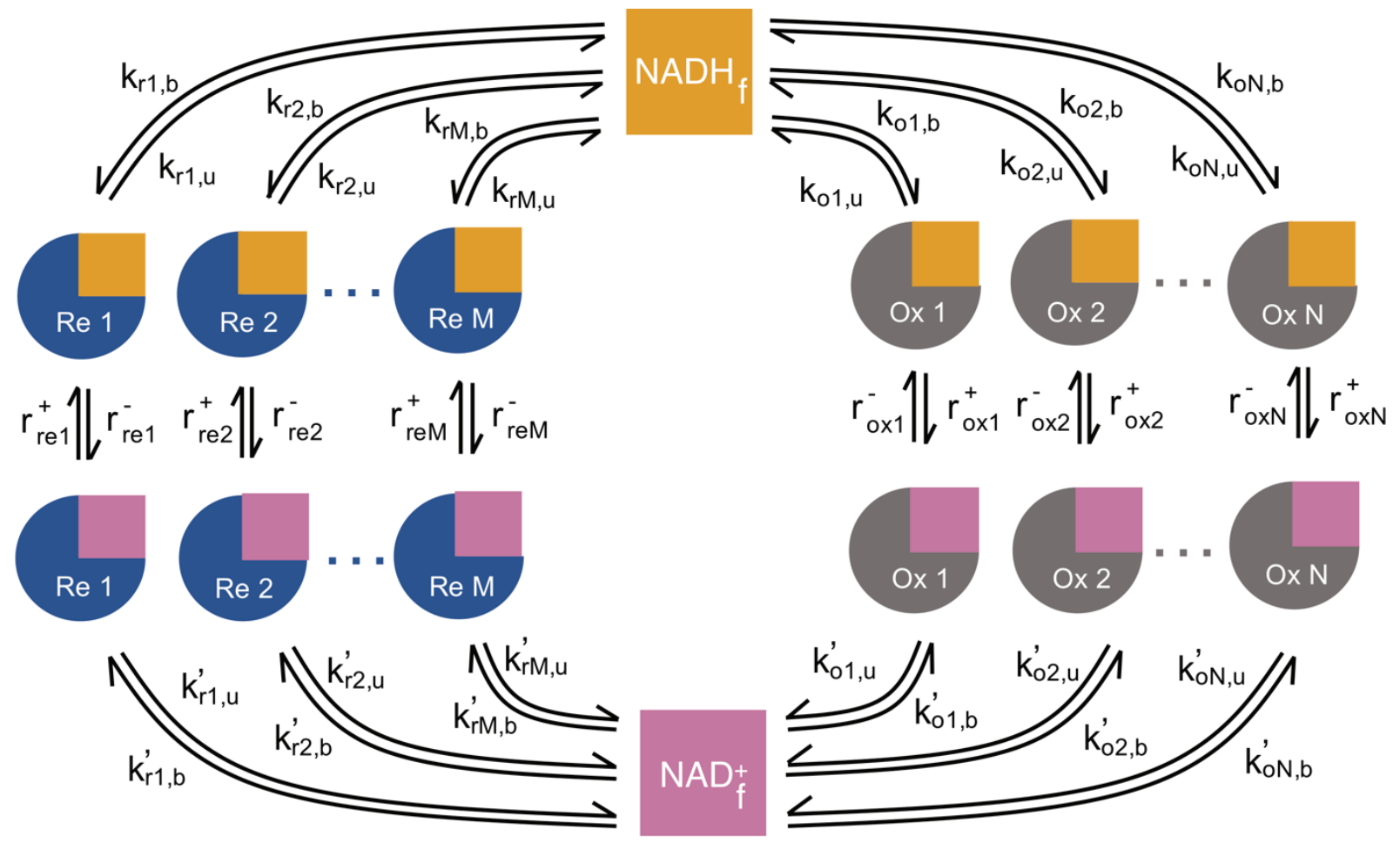

Figure 2 - figure supplement 1 | The detailed NADH redox model. The model consists of redox loops with N oxidases and $\mathrm{M}$ reductases. The binding and unbinding rate of NADH to the $i$ th oxidase (reductase) is $k_{o i, b}$ and $k_{o i, u}$ $\left(k_{r i, b}\right.$ and $\left.k_{r i, u}\right)$. The binding and unbinding rate of $\mathrm{NAD}^{+}$to the $i$ th oxidase (reductase) is $k_{o i, b}^{\prime}$ and $k_{o i, u}^{\prime}\left(k_{r i, b}^{\prime}\right.$ and $k_{r i, u}^{\prime}$ ). Once bound, the forward and reverse reaction rates are $r_{o x i}^{+}$and $r_{o x i}^{-}$for the $i$ th oxidase; $r_{r e i}^{+}$and $r_{r e i}^{-}$for the $i$ th reductase. All rates can be arbitrary functions of metabolite concentrations, enzyme concentrations, and other factors (such as $\mathrm{pH}$ and mitochondrial membrane potential).

We coarse-grain the detailed NADH redox model (Figure 2-figure supplement 1) by coarsegraining all oxidases into a single effective oxidase and all reductases into a single effective reductase (Figure 2b). We relate the kinetic rates of the coarse-grained model to those of the detailed model by preserving the global binding and unbinding fluxes and the global reaction fluxes through the oxidases and reductases.

We first coarse-grain the oxidases and reductases:

$$
[N A D H \cdot o x]=\sum_{i=1}^{N}\left[N A D H \cdot o x_{i}\right], \quad[N A D H \cdot r e]=\sum_{i=1}^{M}\left[N A D H \cdot r e_{i}\right]
$$


We require the global binding and unbinding fluxes of NADH to the effective oxidase and reductase to be equal to the sum of their binding and unbinding fluxes to all of the individual oxidases and reductases:

$$
\begin{gathered}
J_{o x, b}=\left[N A D H_{f}\right] \sum_{i=1}^{N} k_{o i, b}=k_{o, b}\left[N A D H_{f}\right](S 6), \\
J_{o x, u}=\sum_{i=1}^{N} k_{o i, u}\left[N A D H \cdot o x_{i}\right]=k_{o, u}[N A D H \cdot o x](S 7), \\
J_{r e, b}=\left[N A D H_{f}\right] \sum_{i=1}^{M} k_{r i, b}=k_{r, b}\left[N A D H_{f}\right](S 8), \\
J_{r e, u}=\sum_{i=1}^{M} k_{r i, u}\left[N A D H \cdot r e_{i}\right]=k_{r, u}[N A D H \cdot r e](S 9),
\end{gathered}
$$

which leads to

$$
\begin{gathered}
k_{o, b}=\sum_{i=1}^{N} k_{o i, b}, \quad k_{r, b}=\sum_{i=1}^{M} k_{r i, b}(S 10), \\
k_{o, u}=\sum_{i=1}^{N} k_{o i, u} \frac{\left[N A D H \cdot o x_{i}\right]}{[N A D H \cdot o x]}, \quad k_{r, u}=\sum_{i=1}^{M} k_{r i, u} \frac{\left[N A D H \cdot r e_{i}\right]}{[N A D H \cdot r e]}(S 11) .
\end{gathered}
$$

We require the global forward and reverse reaction flux through the effective oxidase and reductase to be equal to the sum of the reaction fluxes through all of the individual oxidases and reductases:

which leads to

$$
\begin{aligned}
& J_{o x}^{+}=\sum_{i=1}^{N} r_{o x i}^{+}\left[N A D H \cdot o x_{i}\right]=r_{o x}^{+}[N A D H \cdot o x](S 12), \\
& J_{o x}^{-}=\sum_{i=1}^{N} r_{o x i}^{-}\left[N A D^{+} \cdot o x_{i}\right]=r_{o x}^{-}\left[N A D^{+} \cdot o x\right](S 13),
\end{aligned}
$$

$$
\begin{aligned}
r_{o x}^{+} & =\sum_{i=1}^{N} r_{o x i}^{+} \frac{\left[N A D H \cdot o x_{i}\right]}{[N A D H \cdot o x]} \\
r_{o x}^{-} & =\sum_{i=1}^{N} r_{o x i}^{-} \frac{\left[N A D^{+} \cdot o x_{i}\right]}{\left[N A D^{+} \cdot o x\right]}
\end{aligned}
$$

By applying the same procedure to $N A D^{+}$, we can obtain the effective reduction rates $r_{r e}^{+}, r_{o x}^{-}$and the effective binding and unbinding rates of $N A D^{+}: k_{o, b}^{\prime}, k_{r, b}^{\prime}, k_{o, u}^{\prime}, k_{r, u}^{\prime}$. We omit the derivation here because these rates are not needed to infer ETC flux. We hence explicitly related the kinetic 
rates of the coarse-grained model (Figure 2b) to those of the detailed model (Figure 2-figure supplement 1).

\section{Appendix 3}

\section{Predicting the ETC flux using the coarse-grained NADH redox model}

\section{The coarse-grained NADH redox model}

We start with the equations characterizing the dynamics of the coarse-grained NADH redox model as described in Figure 2b:

$$
\begin{aligned}
& \frac{d[N A D H \cdot r e]}{d t}=k_{r, b}\left[N A D H_{f}\right]-k_{r, u}[N A D H \cdot r e]+r_{r e}^{+}\left[N A D^{+} \cdot r e\right]-r_{r e}^{-}[N A D H \cdot r e](\mathrm{S} 16), \\
& \frac{d\left[N A D H_{f}\right]}{d t}=k_{r, u}[N A D H \cdot r e]+k_{o, u}[N A D H \cdot o x]-k_{r, b}\left[N A D H_{f}\right]-k_{o, b}\left[N A D H_{f}\right](\mathrm{S} 17), \\
& \frac{d[N A D H \cdot o x]}{d t}=k_{o, b}\left[N A D H_{f}\right]-k_{o, u}[N A D H \cdot o x]-r_{o x}^{+}[N A D H \cdot o x]+r_{o x}^{-}\left[N A D^{+} \cdot o x\right](\mathrm{S} 18), \\
& \frac{d\left[N A D^{+} \cdot o x\right]}{d t}=k_{o, b}^{\prime}\left[N A D_{f}^{+}\right]-k_{o, u}^{\prime}\left[N A D^{+} \cdot o x\right]+r_{o x}^{+}[N A D H \cdot o x]-r_{o x}^{-}\left[N A D^{+} \cdot o x\right](\mathrm{S} 19), \\
& \frac{d\left[N A D_{f}^{+}\right]}{d t}=k_{r, u}^{\prime}\left[N A D^{+} \cdot r e\right]+k_{o, u}^{\prime}\left[N A D^{+} \cdot o x\right]-k_{r, b}^{\prime}\left[N A D_{f}^{+}\right]-k_{o, b}^{\prime}\left[N A D_{f}^{+}\right](\mathrm{S} 20), \\
& \frac{d\left[N A D^{+} \cdot r e\right]}{d t}=k_{r, b}^{\prime}\left[N A D_{f}^{+}\right]-k_{r, u}^{\prime}\left[N A D^{+} \cdot r e\right]-r_{r e}^{+}\left[N A D^{+} \cdot r e\right]+r_{r e}^{-}[N A D H \cdot r e](\mathrm{S} 21),
\end{aligned}
$$

where $\left[N A D H_{f}\right]$ and $\left[N A D_{f}^{+}\right]$are the concentrations of free $\mathrm{NADH}$ and free $\mathrm{NAD}^{+} ;[N A D H \cdot r e]$ and $\left[N A D^{+} \cdot r e\right]$ are concentrations of reductase-bound $\mathrm{NADH}$ and $\mathrm{NAD}^{+} ;[N A D H \cdot o x]$ and $\left[N A D^{+} \cdot o x\right]$ are concentrations of oxidase-bound $\mathrm{NADH}$ and $\mathrm{NAD}^{+} ; k$ denotes binding $(b)$ and unbinding $(u)$ rates, with subscript $r$ and $o$ denoting reductase and oxidase, respectively; $r_{r e}^{+}$and $r_{r e}^{-}$are the forward and reverse reaction rates of the reductase; $r_{o x}^{+}$and $r_{o x}^{-}$are the forward and reverse reaction rates of the oxidase. The reaction rates, and binding and unbinding rates, can be arbitrary functions of metabolite concentrations, enzyme concentrations, and other variables (such as membrane potential, oxygen concentration, and the presence of added drugs).

\section{Predicting the ETC flux}

The flux through the ETC is

$$
J_{o x} \equiv r_{o x}^{+}[N A D H \cdot o x]-r_{o x}^{-}\left[N A D^{+} \cdot o x\right](S 22)
$$

At steady state (or in the quasistatic limit), all the time derivatives are zero. Setting $d[N A D H \cdot o x] / d t$ (equation (S18)) to zero, we obtained the steady state flux through the ETC:

$$
J_{o x}=k_{o, b}\left[N A D H_{f}\right]-k_{o, u}[N A D H \cdot o x](S 23) \text {. }
$$

Setting $d\left[N A D H_{f}\right] / d t$ (eqn. (S17)) to zero gives: 


$$
\left(k_{o, b}+k_{r, b}\right)\left[N A D H_{f}\right]=k_{o, u}[N A D H \cdot o x]+k_{r, u}[N A D H \cdot r e](S 24)
$$

and using:

$$
[N A D H \cdot r e]+[N A D H \cdot o x]=\left[N A D H_{b}\right](S 25)
$$

from which we solved for $[N A D H \cdot o x]$ :

$$
[N A D H \cdot o x]=\frac{k_{r, b}+k_{o, b}}{k_{o, u}-k_{r, u}}\left[N A D H_{f}\right]-\frac{k_{r, u}}{k_{o, u}-k_{r, u}}\left[N A D H_{b}\right](S 26) .
$$

Substituting $[N A D H \cdot o x]$ in equation (S23) with equation (S26), we obtained our central result:

$$
J_{o x}=\tilde{r}_{o x}\left[N A D H_{f}\right](\mathrm{S} 27)
$$

From equation (S27) we see that the flux through ETC is a product of the turnover rate of free $\mathrm{NADH}, \tilde{r}_{o x}$, and the concentration of free NADH, $\left[N A D H_{f}\right]$, where

and

$$
\tilde{r}_{o x}=\alpha\left(\beta-\beta_{e q}\right)(\mathrm{S} 28)
$$

$$
\alpha=\frac{k_{o, u} k_{r, u}}{k_{o, u}-k_{r, u}}(S 29),
$$

where we defined the NADH bound ratio and its equilibrium counterpart as:

$$
\begin{gathered}
\beta=\frac{\left[N A D H_{b}\right]}{\left[N A D H_{f}\right]}(S 30), \\
\beta_{e q}=\beta_{e q}^{o x}+\beta_{e q}^{r e}(S 31), \\
\beta_{e q}^{o x}=\frac{k_{o, b}}{k_{o, u}}(S 32), \\
\beta_{e q}^{r e}=\frac{k_{r, b}}{k_{r, u}}(S 33) .
\end{gathered}
$$




\section{Appendix 4}

\section{Connecting the NADH redox model to detailed biophysical models of mitochondrial metabolism}

In this section, we show that the coarse-grained NADH redox model described above, and in Figure $2 \mathrm{~b}$ of the main text, can be directly related to detailed biophysical models of mitochondrial metabolism, including previously published models (Beard, 2005; Korzeniewski and Zoladz, 2001; Hill, 1977; Jin and Bethke 2002; Chang et al., 2011).

In mitochondria, NADH oxidation is catalyzed by complex I of the electron transport chain, which has the overall reaction:

$$
H^{+}+N A D H+Q \rightleftarrows N A D^{+}+Q H_{2}+4 \Delta H^{+}(S 34),
$$

where two electrons are transferred from NADH to ubiquinone Q, and 4 protons are pumped out of the mitochondrial matrix. To connect our model with detailed model of complex I, we rewrite the flux through the ETC:

$$
J_{o x}=r_{o x}^{+}[N A D H \cdot o x]-r_{o x}^{-}\left[N A D^{+} \cdot o x\right](\mathrm{S} 35),
$$

Using

$$
\begin{aligned}
& {[N A D H \cdot o x]=\alpha\left(\frac{\beta_{e q}^{r e}}{k_{o, u}}+\frac{\beta_{e q}^{o x}}{k_{r, u}}-\frac{\beta}{k_{o, u}}\right)\left[N A D H_{f}\right](\mathrm{S} 36),} \\
& {\left[N A D^{+} \cdot o x\right]=\alpha^{\prime}\left(\frac{\beta_{e q}^{\prime r e}}{k_{o, u}^{\prime}}+\frac{\beta_{e q}^{\prime o x}}{k_{r, u}^{\prime}}-\frac{\beta^{\prime}}{k_{o, u}^{\prime}}\right)\left[N A D_{f}^{+}\right](\mathrm{S} 37),}
\end{aligned}
$$

where

$$
\begin{gathered}
\alpha=\frac{k_{o, u} k_{r, u}}{k_{o, u}-k_{r, u}}, \beta=\frac{\left[N A D H_{b}\right]}{\left[N A D H_{f}\right]}, \quad \beta_{e q}^{o x}+\beta_{e q}^{r e}=\beta_{e q}, \quad \beta_{e q}^{o x}=\frac{k_{o, b}}{k_{o, u}}, \quad \beta_{e q}^{r e}=\frac{k_{r, b}}{k_{r, u}}(\mathrm{~S} 38) \\
\alpha^{\prime}=\frac{k_{o, u}^{\prime} k_{r, u}^{\prime}}{k_{o, u}^{\prime}-k_{r, u}^{\prime}}, \beta^{\prime}=\frac{\left[N A D_{b}^{+}\right]}{\left[N A D_{f}^{+}\right]}, \quad \beta_{e q}^{\prime o x}+\beta_{e q}^{\prime r e}=\beta_{e q}^{\prime}, \quad \beta_{e q}^{\prime o x}=\frac{k_{o, b}^{\prime}}{k_{o, u}^{\prime}}, \quad \beta_{e q}^{\prime r e}=\frac{k_{r, b}^{\prime}}{k_{r, u}^{\prime}}(\mathrm{S} 39)
\end{gathered}
$$


as

$$
J_{o x}=\tilde{r}_{o x}^{+}\left[N A D H_{f}\right]-\tilde{r}_{o x}^{-}\left[N A D_{f}^{+}\right]=\tilde{r}_{o x}\left[N A D H_{f}\right](\mathrm{S} 40),
$$

where

$$
\begin{gathered}
\tilde{r}_{o x}^{+}=\alpha\left(\frac{\beta_{e q}^{r e}}{k_{o, u}}+\frac{\beta_{e q}^{o x}}{k_{r, u}}-\frac{\beta}{k_{o, u}}\right) r_{o x}^{+}(\mathrm{S} 41), \\
\tilde{r}_{o x}^{-}=\alpha^{\prime}\left(\frac{\beta_{e q}^{r e}}{k_{o, u}^{\prime}}+\frac{\beta_{e q}^{\prime o x}}{k_{r, u}^{\prime}}-\frac{\beta^{\prime}}{k_{o, u}^{\prime}}\right) r_{o x}^{-}(\mathrm{S} 42), \\
\tilde{r}_{o x}=\alpha\left(\beta-\beta_{e q}\right)=\left(\tilde{r}_{o x}^{+}+\tilde{r}_{o x}^{-} \frac{1+\beta}{1+\beta^{\prime}}\right)-\frac{N}{\left[N A D H_{f}\right]} \tilde{r}_{o x}^{-}(S 43) .
\end{gathered}
$$

The last equality in Equation (S43) is obtained by assuming that the total concentration of NADH plus $\mathrm{NAD}^{+}$is constant:

$$
N=\left[N A D H_{f}\right]+\left[N A D_{f}^{+}\right]+\left[N A D H_{b}\right]+\left[N A D_{b}^{+}\right](S 44) .
$$

Equation (S40) allows us to connect our coarse-grained model to previously published detailed

\begin{tabular}{|c|c|c|c|c|}
\hline Model & Flux & $\tilde{r}_{o x}^{+}$ & $\tilde{r}_{o x}^{-}$ & $\tilde{r}_{o x}$ \\
\hline Beard et al., 2019 & $J_{C I}=\tilde{r}_{o x}^{+}\left[N A D H_{f}\right]-\tilde{r}_{o x}^{-}\left[N A D_{f}^{+}\right]$ & $\tilde{r}_{o x}^{+}=X_{C 1} e^{\frac{\Delta \tilde{G}_{C I}}{R T}}$ & $\tilde{r}_{o x}^{-}=X_{C 1}$ & $\tilde{r}_{o x}=\left(\tilde{r}_{o x}^{+}+\tilde{r}_{o x}^{-} \frac{1+\beta}{1+\beta^{\prime}}\right)-\frac{N}{\left[N A D H_{f}\right]} \tilde{r o x}_{o x}^{-}$ \\
\hline $\begin{array}{l}\text { Chang et al., } 2011 \\
\text { Jin et al., } 2002\end{array}$ & $J_{C I}=\tilde{r}_{o x}\left[N A D H_{f}\right]$ & N/A & N/A & $\begin{array}{c}\tilde{r}_{o x}=V_{\max }\left(\frac{\left[N_{T}\right]}{\left[N_{T}\right]+K_{S, D}}\right)\left(\frac{\left[Q_{T}\right]}{\left[Q_{T}\right]+K_{S, A}}\right) \times \\
\left(\frac{[Q]}{[Q]+\left[Q H_{2}\right] K_{R, A}}\right)\left(\frac{1}{\left[N A D H_{f}\right]+\left[N A D_{f}^{+}\right] K_{R, D}}\right) \times \\
\left(1-e^{-\frac{\Delta G_{C I}}{R T}}\right)\end{array}$ \\
\hline Hill, 1977 & $J_{C I}=J_{\max }\left(1-e^{\frac{-\Delta G_{C I}}{R T}}\right)$ & N/A & N/A & $\tilde{r}_{o x}=J_{\max }\left(1-e^{\frac{-\Delta G_{C I}}{R T}}\right) /\left[N A D H_{f}\right]$ \\
\hline $\begin{array}{l}\text { Korzeniewski } \\
\text { and Zoladz, } 2001\end{array}$ & $J_{C I}=k_{C I} \Delta G_{C I}$ & N/A & $\mathrm{N} / \mathrm{A}$ & $\tilde{r}_{o x}=k_{C I} \Delta G_{C I} /\left[N A D H_{f}\right]$ \\
\hline \multicolumn{5}{|c|}{$\begin{array}{l}\Delta G_{C I}=-\left[\Delta G_{0, C I}+4 \Delta G_{H}-R T \ln \left(\frac{\left[H^{+}\right]}{10^{-7}}\right)-R T \ln \left(\frac{[Q]}{\left[Q H_{2}\right]}\right)-R T \ln \left(\frac{\left[N A D H_{f}\right]}{\left[N A D_{f}^{+}\right]}\right)\right] ; \Delta \tilde{G}_{C I}=\Delta G_{C I}-R T \ln \left(\frac{\left[N A D H_{f}\right]}{\left[N A D_{f}^{+}\right]}\right) ; \Delta G_{0, C I}=-69.37 \mathrm{~kJ} / \mathrm{mol} \\
{\left[N_{T}\right]=\left[N A D H_{f}\right]+\left[N A D_{f}^{+}\right],\left[Q_{T}\right]=[Q]+\left[Q H_{2}\right]}\end{array}$} \\
\hline
\end{tabular}
models of complex I. By equating the flux through complex I, $J_{C 1}$, in previous models to the flux through the ETC in our NADH redox model, $J_{o x}$, we can determine $\tilde{r}_{o x}$ (and $\tilde{r}_{o x}^{+}$and $\tilde{r}_{o x}^{-}$) in terms of variables defined in those more detailed models. In Table 1, we summarize the relationship between the NADH redox model and several previously published models of complex I.

Table 1 | Connection of the NADH redox model to detailed models of complex I. $\Delta G_{H}$ is the proton motive force. $\Delta G_{C I}$ is the free energy difference of the reaction at complex I. $\Delta G_{0, C I}$ is the standard free energy difference of the reaction at complex I. 


\section{Appendix 5}

\section{Flux inference procedures using the coarse-grained NADH redox model}

Equation (S27), or equivalently equation 1 from the main text, can be used to infer the flux through the ETC, $J_{o x}$, from FLIM measurements of NADH across a wide range of metabolic perturbations (Figure 3-figure supplement 1). To do so, we infer the turnover rate of free NADH, $\tilde{r}_{o x}$, and the concentration of free NADH, $\left[N A D H_{f}\right]$. The product of $\tilde{r}_{o x}$ and $\left[N A D H_{f}\right]$ gives $J_{o x} \cdot\left[N A D H_{f}\right]$ can be obtained using equation (S2) (Figure 3-figure supplement 1e). In this section, we introduce two procedures to obtain $\tilde{r}_{o x}$ : one from the measurement of NADH bound ratio, and the other from the measurement of NADH long fluorescence lifetime.

\section{Inferring $\tilde{r}_{o x}$ from NADH bound ratio $\beta$}

From equation (S28), $\tilde{r}_{o x}=\alpha\left(\beta-\beta_{e q}\right)$. We measure the NADH bound ratio, $\beta$, using $\beta=$ $f /(1-f)$, where $f$ is the NADH bound fraction obtained by fitting the fluorescence decay curve of NADH (See Methods). We obtain the equilibrium bound ratio, $\beta_{e q}$, by dropping the oxygen level to the lowest achievable value with our setup, $\left[\mathrm{O}_{2}\right]=0.26 \pm 0.04 \mu \mathrm{M}$ in our experiments. Note that $\beta_{e q}$ changes with culture conditions, and therefore needs to be separately determined for every drug and nutrient perturbation (Figure 3-figure supplement 1h). We obtain $\alpha$ using direct measurement of $J_{o x}$ from oxygen consumption rate (OCR) measurements:

$$
\alpha=\frac{J_{o x}}{\left(\beta-\beta_{e q}\right)\left[N A D H_{f}\right]}=2 \frac{O C R}{\left(\beta-\beta_{e q}\right)\left[N A D H_{f}\right] V_{m}}(S 45),
$$

where $V_{m}=9.5 \times 10^{4} \mu \mathrm{m}^{3}$ is the average volume of mitochondria per oocyte approximated from the area fraction of mitochondria based on the segmentation, where the mitochondrial area fraction is estimated at $46 \%$ and oocyte volume at $2 \times 10^{5} \mu \mathrm{m}^{3}$. Using $O C R=2.68 \pm 0.06 \mathrm{fmol} / \mathrm{s}$ per oocyte in the AKSOM media at $50 \mu \mathrm{M}$ oxygen level, we get $\alpha=5.4 \pm 0.2 s^{-1} . \alpha$ is approximated as a constant that does not vary with drug and nutrient perturbations.

Once $\alpha$ is calibrated for one condition using equation (S45), and $\beta_{e q}$ is determined from an oxygen drop experiment, then subsequent FLIM measurements of $\beta$ and $\left[N A D H_{f}\right]$ can be used with equation (S27) to determine the absolute value of $\tilde{r}_{o x}$ (Figure 4-figure supplement 1) and $J_{o x}$ (Figure 4) for all conditions. 

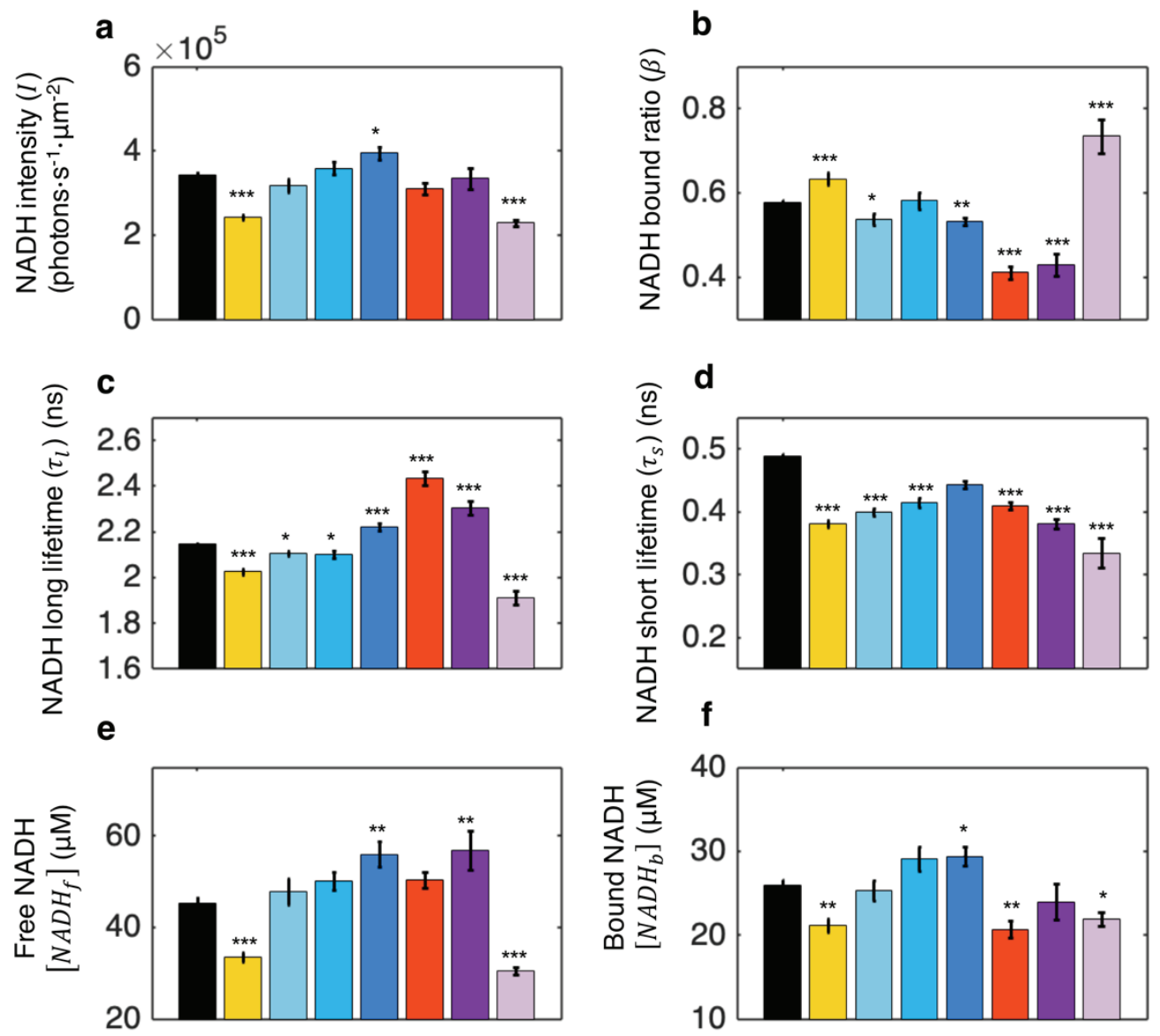

g

$$
\text { h }
$$
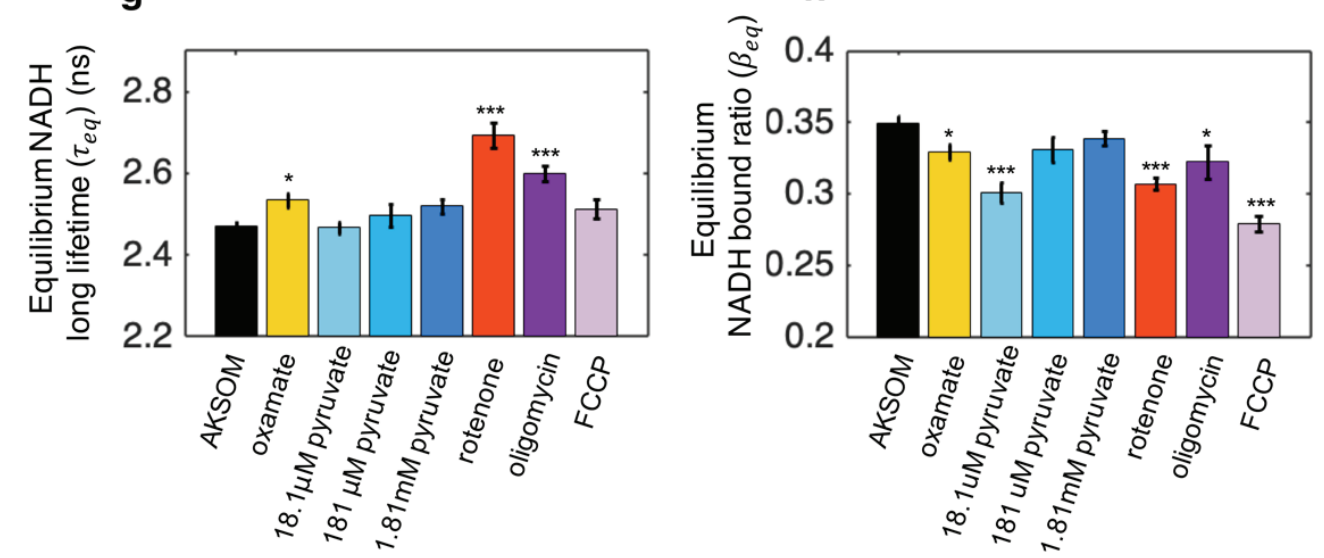

Figure 3-figure supplement 1 | FLIM measurements of NADH in mitochondria for different biochemical perturbations. a, NADH intensity. b-d, NADH bound ratio and NADH long and short fluorescence lifetimes obtained from fitting FLIM decay curves using the two-exponential decay model (Method). e-f, free and bound NADH concentrations obtained by using equations (S2) and (S3), and the calibration from equation (S4). g-h, Equilibrium NADH long lifetime and bound ratio, measured at the lowest oxygen level under different conditions. 9mM sodium oxamate was present in all conditions except for AKSOM to suppress the cytoplasmic signal and allow for better mitochondrial segmentation. AKSOM $(n=68)$, oxamate $(n=20)$, pyruvate $(n=10,11,15$, from low to high concentrations), rotenone ( $n=15)$, oligomycin $(n=13)$ and FCCP $(n=15) . n$ is the number of oocytes. Error bars 
represent standard error of the mean (s.e.m). Student's t-test is performed pairwise between perturbations and AKSOM condition. ${ }^{*} \mathrm{p}<0.05, * * \mathrm{p}<0.01,{ }^{* * *} \mathrm{p}<0.001$.

\section{Inferring $\tilde{r}_{O X}$ from NADH long fluorescence lifetime $\tau_{l}$}

In this section, we derive an alternative procedure for determining the turnover rate of free NADH $\tilde{r}_{o x}$, and hence $J_{o x}$ from equation (S27), using changes in the NADH long fluorescence lifetime. The NADH long fluorescence lifetime, $\tau_{l}$, is associated with enzyme-bound NADH (Sharick et al., 2018). In the coarse-grained NADH redox model described above, and in Figure $2 b$, the enzyme-bound NADH consists of reductase-bound NADH $([N A D H \cdot r e])$ and oxidase-bound $\mathrm{NADH}([N A D H \cdot o x])$. We therefore assume that the experimentally measured NADH long lifetime, $\tau_{l}$, is a linear combination of the lifetimes of $[N A D H \cdot o x]$ and $[N A D H \cdot r e]$ :

$$
\tau_{l}=\tau_{o x} \frac{[N A D H \cdot o x]}{[N A D H \cdot o x]+[N A D H \cdot r e]}+\tau_{r e} \frac{[N A D H \cdot r e]}{[N A D H \cdot o x]+[N A D H \cdot r e]}(S 46),
$$

where $\tau_{o x}$ and $\tau_{r e}$ are the fluorescence lifetimes corresponding to the oxidase-bound NADH and reductase-bound $\mathrm{NADH}$, respectively. Substituting $[N A D H \cdot o x]$ and $[N A D H \cdot r e]$ in equation (S46) with $\beta$ using equations (S25)-(S26) and equation (S30), we derive that the NADH long fluorescence lifetime $\tau_{l}$ is linearly related to the inverse of the NADH bound ratio $1 / \beta$ :

with

$$
\tau_{l}=A \frac{1}{\beta}+B(S 47)
$$

$$
\begin{gathered}
A=\left(\tau_{o x}-\tau_{r e}\right) \frac{k_{o, b}+k_{r, b}}{k_{o, u}-k_{r, u}}(S 48), \\
B=\frac{k_{o, u} \tau_{r e}-k_{r, u} \tau_{o x}}{k_{o, u}-k_{r, u}}(S 49) .
\end{gathered}
$$

The predicted linear relationship between $\tau_{l}$ and $1 / \beta$ is empirically observed during oxygen drop experiments, as shown in Figure 3d and Figure 3-figure supplement 2. This self-consistency check argues for the validity of the assumption in equation (S46).

At equilibrium, when there is no flux through the ETC (i.e. $J_{o x}=0$ ), equation $(S 47)$ gives:

$$
\tau_{e q}=A \frac{1}{\beta_{e q}}+B(S 50)
$$

where $\tau_{e q}$ is the NADH long lifetime at equilibrium. Using equations (S47) and (S50) in combination with (S28), we obtain $\tilde{r}_{o x}$ in terms of $\tau_{l}$ :

$$
\tilde{r}_{o x}=\alpha \frac{A}{\tau_{e q}-B}\left(\frac{\tau_{e q}-\tau_{l}}{\tau_{l}-B}\right)(S 51)
$$

where $\mathrm{A}$ and $\mathrm{B}$ are the slope and offset of the linear relation between $\tau_{l}$ and $1 / \beta$ in equation (S47).

We experimentally measure A and B for each oocyte from the slope and offset of a linear fit between $\tau_{l}$ and $1 / \beta$ during oxygen drop experiments across all perturbations (Figure $3 \mathrm{~d}$; Figure 
3-figure supplement 2). We obtain the equilibrium long lifetime, $\tau_{e q}$, by FLIM measurements at the lowest achievable oxygen level, $\left[\mathrm{O}_{2}\right]=0.26 \pm 0.04 \mu \mathrm{M}$ in our experiments. Once $\mathrm{A}, \mathrm{B}$, and $\tau_{e q}$ are measured, equation (S51) can be used to determine $\tilde{r}_{o x}$ from FLIM measurements of $\tau_{l}$. If $\alpha$ is not known, this procedure can only be used to obtain $\tilde{r}_{o x}$ up to a constant of proportionality. If $\alpha$ is independently measured from equation (S45), then equation (S51) can be used to determine the absolute value of $\tilde{r}_{o x}$ (Figure 4-figure supplement 1).

As described in the main text, $\tilde{r}_{o x}$ inferred using equation (S51) produces the same results as $\tilde{r}_{o x}$ inferred using equation (S28) (Figure 3e in the main text). The agreement between these two methods is a strong self-consistency check of the model.
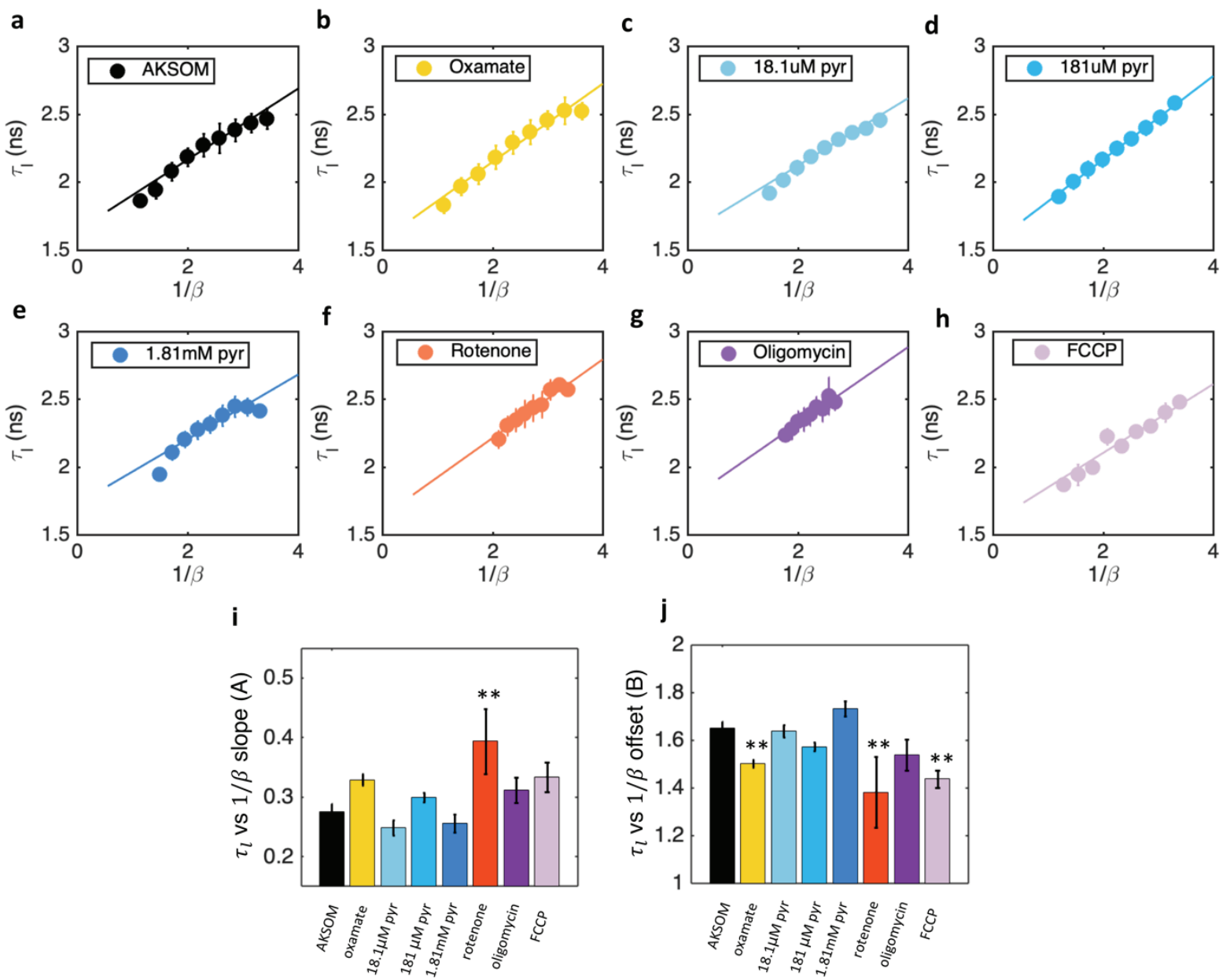

Figure 3-figure supplement 2 | NADH long fluorescence lifetime $\tau_{l}$ is linearly related to the inverse of the NADH bound ratio $1 / \boldsymbol{\beta}$. a-h, $\tau_{l}$ vs $1 / \beta$ during oxygen drop for all perturbations and the corresponding linear fitting. The fitting is performed for each oocyte independently. The plot shows the average fitting across all oocytes. $\mathbf{i}-\mathbf{j}$, slope (A) and offset (B) obtained by fitting equation (S47) to $\tau_{l}$ vs $1 / \beta$. Student's t-test is performed pairwise between perturbations and AKSOM condition. Error bars represent standard error of the mean (s.e.m). ${ }^{*} \mathrm{p}<0.05,{ }^{* *} \mathrm{p}<0.01$, $* * * \mathrm{p}<0.001$. 
a
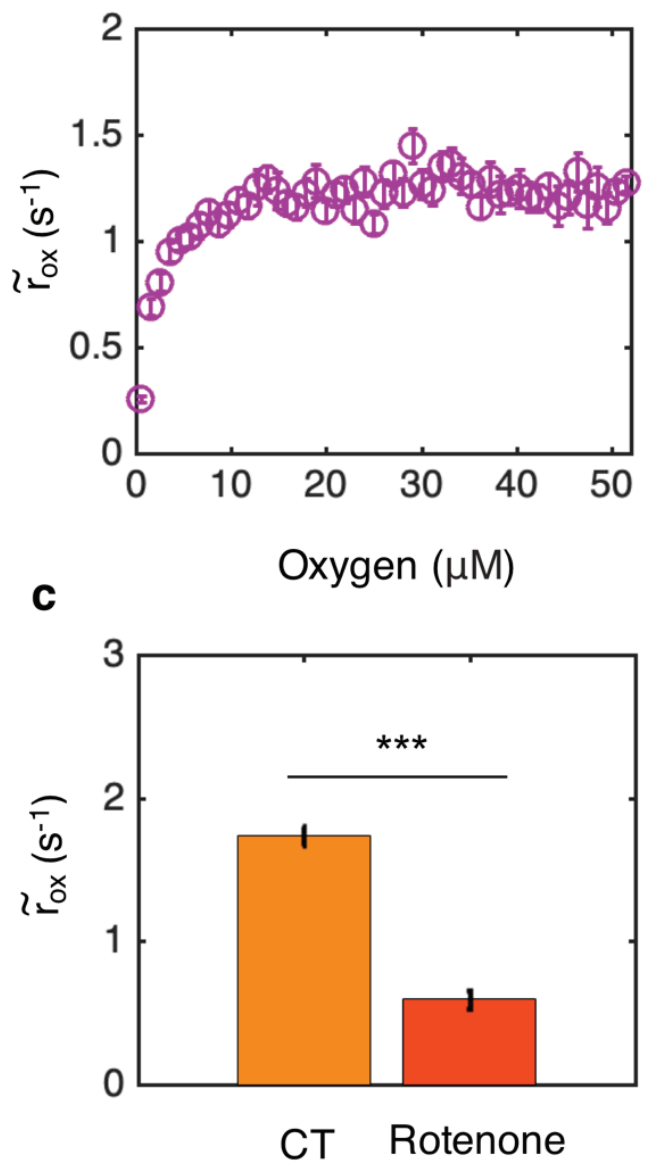

b

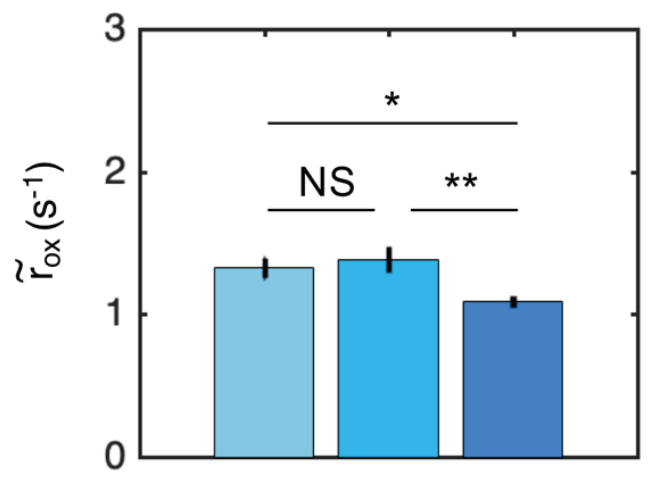

$18.1181 \quad 1810$

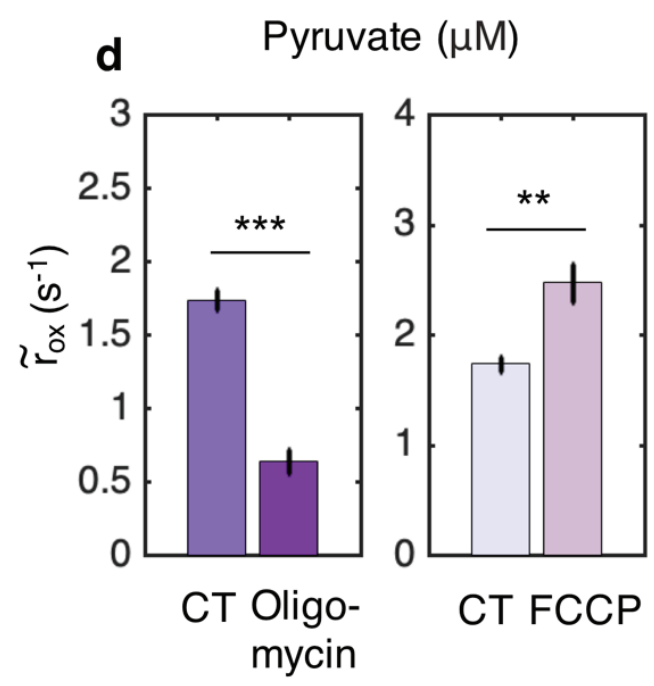

Figure 4-figure supplement 1 | Response of the turnover rate of free NADH to mitochondrial perturbations. ad, turnover rate $\tilde{r}_{o x}$ in response to oxygen ( $\left.\mathrm{n}=68\right)$, pyruvate $(\mathrm{n}=10,11,15$, from low to high concentrations), rotenone $(n=15)$, oligomycin $(n=13)$ and FCCP perturbations $(n=15)$. CT is AKSOM with oxamate $(n=20)$. $n$ is the number of oocytes. Error bars represent standard error of the mean (s.e.m). ${ }^{*} \mathrm{p}<0.05,{ }^{* *} \mathrm{p}<0.01,{ }^{* * *} \mathrm{p}<0.001$. 


\section{Appendix 6}

\section{Spatial gradient of mitochondrial metabolism in mouse oocyte}

\section{Subcellular spatial gradient of mitochondrial membrane potential}

a
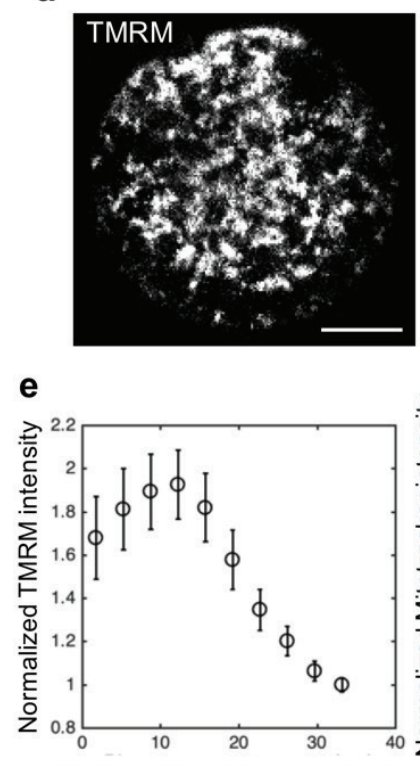

Distance from oocyte center $(\mu \mathrm{m})$ Distance from oocyte center $(\mu \mathrm{m})$
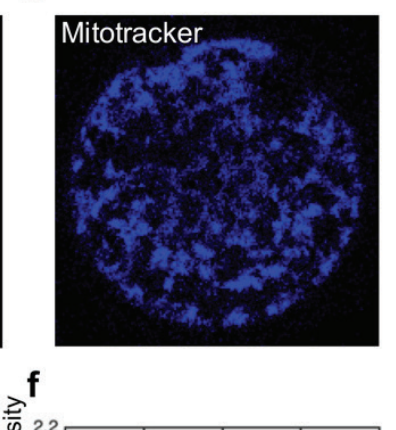

b

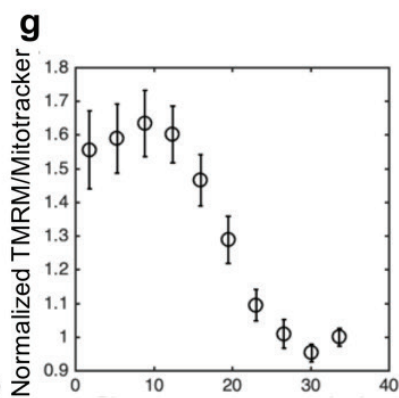

c

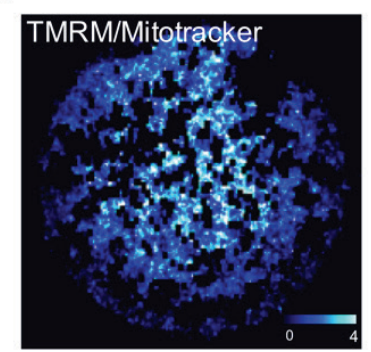

d

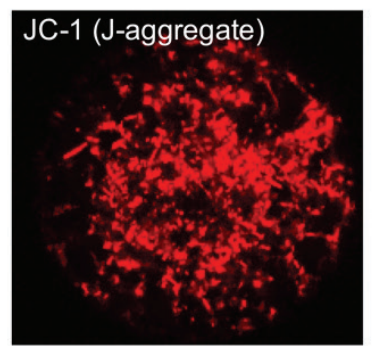

h

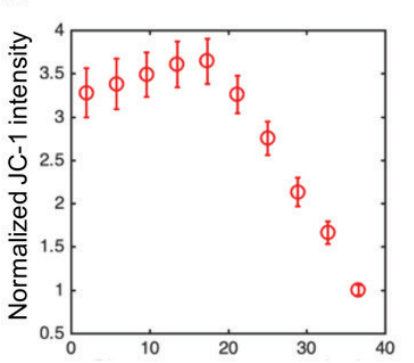

Figure 5-figure supplement 1 | Subcellular spatial gradient of mitochondrial membrane potential. a-d, Intensity images of TMRM, Mitotracker Red FM, TMRM/Mitotracker ratio, JC-1 (J-aggregate). e-h, Normalized subcellular intensity gradient of the corresponding dyes as a function of distance from the oocyte's center ( $\mathrm{n}=18$ for each dye).

As shown in the main text, we observed a strong spatial gradient of the intensity of TMRM in mitochondria in oocytes. TMRM is a potential-sensitive dye that preferentially accumulates in mitochondria with higher membrane potential (Figure $5 \mathrm{~d}$-e in the main text). To test whether this spatial gradient is due to the subcellular variation of mitochondrial membrane potential or the variation in mitochondrial mass, we labelled mitochondria with a potential-insensitive dye Mitotracker Red FM to quantify mitochondrial mass, together with TMRM. We did not observe a strong gradient of mitotracker intensity (Figure 5-figure supplement 1b,f) as compared to TMRM intensity (Figure 5-figure supplement 1a,e) within the same oocyte, indicating the mitochondrial mass is uniformly distributed. We further normalized the TMRM intensity by the mitotracker intensity, and observed a strong spatial gradient of the ratio (Figure 5-figure supplement 1c,g). These results suggest that the spatial gradient of TMRM is due to the variation of mitochondrial membrane potential, rather than the variation of mitochondrial mass. Finally, to test the robustness of the result, we used an alternative potential-sensitive dye JC-1, and observed a similar spatial gradient of mitochondrial membrane potential (Figure 5-figure supplement 1d,h). Taken together, these results show that the subcellular spatial gradient of mitochondrial membrane potential is a robust observation that does not depend on the variation of mitochondrial mass or the type of dye used. 


\section{$\beta_{e q}$ is uniform within the oocyte}

a

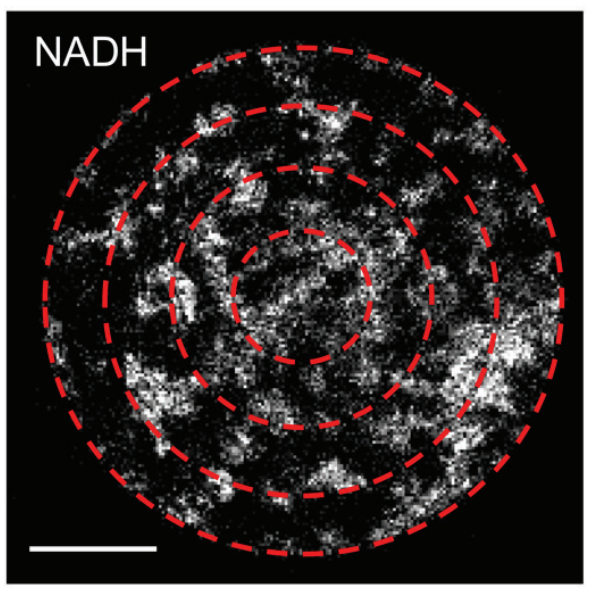

b

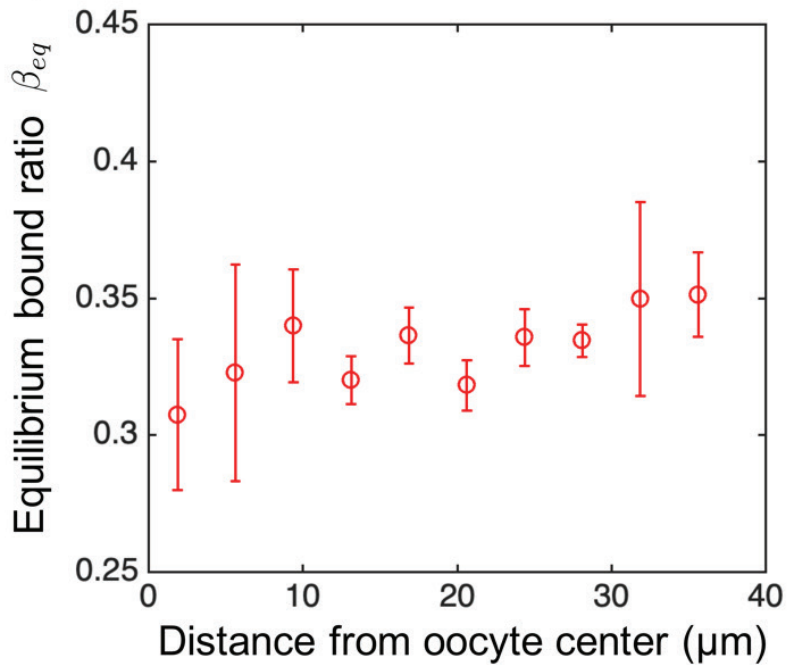

Figure 5-figure supplement $2 \mid \boldsymbol{\beta}_{e q}$ is uniform within the oocyte. a, NADH intensity image with oocyte partitioned by equal-distanced concentric rings. b, Equilibrium bound ratio $\beta_{e q}$ as a function of distance from the oocyte's center obtained by completely inhibiting ETC with rotenone. $(n=10)$.

To obtain subcellular ETC flux as a function of distance to the oocyte's center using Equation 1 in the main text, we need to know the spatial variation of $\beta_{e q}$. While the NADH bound ratio at the lowest oxygen level gives a good approximation for the average $\beta_{e q}$ of the cell (Figure 3-figure supplement 1h), subpopulations of mitochondria closer to the cell membrane are exposed to slightly higher oxygen level than those away from the cell membrane, obscuring the determination of the spatial variation of $\beta_{e q}$. Hence to obtain the spatial variation of $\beta_{e q}$ throughout the oocyte, we inhibited the ETC completely using $15 \mu \mathrm{M}$ of sodium rotenone, an inhibitor of complex I in the ETC, for an extended period of time until the NADH bound ratio reaches the lowest level. We then fit the NADH decay curves from mitochondrial pixels within equal-distanced concentric rings (Figure 5-figure supplement 2a) to obtain $\beta_{e q}$ as a function of distance from the oocyte's center (Figure 5-figure supplement $2 \mathrm{~b}$ ). The resulting $\beta_{e q}$ is uniform throughout the oocyte and is equal to the average $\beta_{e q}$ obtained by fitting the decay curve from all mitochondrial pixels in the oocyte at the lowest oxygen level (Figure 3-figure supplement 1h). Therefore, we used the average $\beta_{e q}$ at the lowest oxygen level to compute the subcellular ETC flux (Figure 5f). 


\section{References}

1. AL-Zubaidi, U., Liu, J., Cinar, O., Robker, R.L., Adhikari, D., Carroll, J., The spatio-temporal dynamics of mitochondrial membrane potential during oocyte maturation, Molecular Human Reproduction, 25(11):695-705(2019)

2. Aryaman, J., Johnston I. G., Jones, N.S., Mitochondrial Heterogeneity, Front. Genet., 25 January 2019, 9,718

3. Beard D.A., A biophysical model of the mitochondrial respiratory system and oxidative phosphorylation. PLoS Comput Biol. 2005. 1(4): e36

4. Becker, W., Fluorescence lifetime imaging-techniques and applications. Journal of Microscopy, 2012. 247: p.119-136.

5. Becker, W., The bh TCSPC Handbook, $8^{\text {th }}$ Edition, 2019

6. Berg, J., Hung, Y. P., Yellen, G., A genetically encoded fluorescent reporter of ATP:ADP ratio. Nat. Methods. 6, 161-166 (2009).

7. Berg, S., Kutra, D., Kroeger, T., Straehle, C.N., Kausler, B.X., Haubold, C., Schiegg, M., Ales, J., Beier, T., Rudy, M., Eren, K., Cervantes, J.I., Xu, B., Beuttenmueller, F., Wolny, A., Zhang, C., Koethe, U., Hamprecht, F.A., Kreshuk, A., ilastik: interactive machine learning for (bio)image analysis, Nature Methods, 16, 1226-1232(2019)

8. Biggers, J.D., Racowsky, C., The development of fertilized human ova to the blastocyst stage in KSOMAA medium: is a two-step protocol necessary? Reprod Biomed Online. 2002 Sep-Oct;5(2):13340

9. Bird, D.K., Yan, L., Vrotsos, K.M., Eliceiri, K.W., Vaughan, E.M., Keely, P.J., White, J.G., Ramanujam, N., Metabolic Mapping of MCF10A Human Breast Cells via Multiphoton Fluorescence Lifetime Imaging of the Coenzyme NADH, Cancer Res 2005 65:8766-8773

10. Blerkom, J.V., Mitochondrial function in the human oocyte and embryo and their role in developmental competence. Mitochandria, 2011. 11: p. 797-813.

11. Bratic, A., Larsson, N., The role of mitochondria in aging, Journal of Clinical Investigation, 2013, 123 : 951-957

12. Brand, M.D., Nicholls, D.G., Assessing mitochondrial dysfunction in cells. Biochem J. 2011. 435(Pt 2): p.297-312

13. Bustamante, S., Jayasena, T., Richani, D., Gilchrist, R.B., Wu, L.E., Sinclair, D.A., Sachdev, P.S., Braidy, N., Quantifying the cellular NAD + metabolome using a tandem liquid chromatography mass spectrometry approach, Metabolomics. 2017 Dec 23; 14(1):15

14. Chang, I., Heiske, M., Letellier, T., Wallace, D., Baldi, P., Modeling of Mitochondria Bioenergetics Using a Composable Chemiosmotic Energy Transduction Rate Law: Theory and Experimental Validation, PLoS One. 2011;6(9):e14820

15. Díaz-García, C.M., Mongeon, R., Lahmann, C., Koveal, D., Zucker, H., Yellen, G., Neuronal stimulation triggers neuronal glycolysis and not lactate uptake, Cell Metab. 2017 Aug 1; 26(2): 361374.e4

16. Díaz-García, C.M., Meyer, D.J., Nathwani, N., Rahman, M., Martinez-Francois, J.R., Yellen, G., The distinct roles of calcium in rapid control of neuronal glycolysis and the tricarboxylic acid cycle, eLife 2021; 10:e64821

17. Dumollard, R., Duchen, M., Carroll, J., The role of mitochondrial function in the oocyte and embryo. Curr Top Dev Biol, 2007. 77: p. 21-49.

18. Ferrick DA, Neilson A, Beeson C. Advances in measuring cellular bioenergetics using extracellular flux. Drug Discov Today. 2008; 13:268-274.

19. Heikal, A.A., Intracellular coenzymes as natural biomarkers for metabolic activities and mitochondrial anomalies. Biomark Med, 2010. 4(2): p. 241-63.

20. Hill, T.L., Free Energy Transduction in Biology, (1977) Academic 
21. Houghton, F.D., Thompson, J.G., Kennedy, C.J., Leese, H.J., Oxygen consumption and energy metabolism of the early mouse embryo. Mol Reprod Dev. 1996.44(4):476-85

22. Imamura, H., Huynh Nhat, K.P., Togawa, H., Saito, K., Lino, R., Kato-Yamada, Y., Nagai, T., Noji, $\mathrm{H}$., Visualization of ATP levels inside single living cells with fluorescence resonance energy transferbased genetically encoded indicators. Proc Natl Acad Sci USA. 2009. 106(37): p. 15651-15656

23. Jin, Q., Bethke, C.M., Kinetics of Electron Transfer through the Respiratory Chain, Biophysical Journal, Volume 83, October 2002, 1797-1808

24. Korzeniewski, B., Zoladz, J.A., A model of oxidative phosphorylation in mammalian skeletal muscle, Biophysical Chemistry, 2001, 92, 17-34

25. Lane, M., Gardner, D.K., Lactate Regulates Pyruvate Uptake and Metabolism in the Preimplantation Mouse Embryo, Biology of Reproduction, Volume 62, Issue 1, 1 January 2000, Pages 16-22

26. Lin, M.T., Flint Beal, M., Mitochondrial dysfunction and oxidative stress in neurodegenerative diseases, Nature, volume 443, pages 787-795 (2006)

27. Liu, Z., Pouli, D., Alonzo, C.A., Varone, A., Karaliota, S., Quinn, K.P., Munger, K., Karalis, K.P., Georgakoudi, I., Mapping metabolic changes by noninvasive, multiparametric, high-resolution imaging using endogenous contrast, Science Advances 07 Mar 2018:Vol. 4, no. 3, eaap9302

28. Lopes, A.S., Larsen, L.H., Ramsing, N., Lovendahl, P., Raty, M., Peippo, J., Greve, T., Callesen, H., Respiration rates of individual bovine in vitro-produced embryos measured with a novel, non-invasive and highly sensitive microsensor system. Reproduction 2005, 130, 669-679

29. Lowell, B.B., Shulman, G.I., Mitochondrial Dysfunction and Type 2 Diabetes, Science 21 Jan 2005: Vol. 307, Issue 5708, pp. 384-387

30. Ma, N., Reyes de Mochel, N., Pham, P.D., Yoo, T.Y., Cho, K.W.Y., Digman, M.A., Label-free assessment of preimplantation embryo quality by the Fluorescence Lifetime Imaging Microscopy (FLIM)-phasor approach. Scientific Reports 2019, 9, 13206

31. Martin, A.S., Ceballo, S., Baeza-Lehnert, F., Lerchundi, R., Valdebenito, R., Contreras-Baeza, Y., Alegria, K., Barros, L.F., Imaging Mitochondrial flux in single cells with a FRET sensor for pyruvate, PloS ONE, 2014, 9(1): e85780

32. Mick, E., Titov, D.V., Skinner, O.S., Sharma, R., Jourdain, A.A., Mootha, V.K., Distinct mitochondrial defects trigger the integrated stress response depending on the metabolic state of the cell, eLife, 2020;9:e49178

33. Mitchell, P., Coupling of Phosphorylation to Electron and Hydrogen Transfer by a Chemi-Osmotic type of Mechanism, Nature 191, 144-148 (1961)

34. Papagiannakis, A., Niebel, B., Wit, E.C., Heinemann, M., Autonomous Metabolic Oscillations Robustly Gate the Early and Late Cell Cycle, Mol Cell. 2017 Jan 19;65(2):285-295.

35. Park, J.O., Rubin S.A., Xu, Y.F., Amador-Noguez, D., Fan, J., Shlomi, T., Rabinowitz, J.D., Metabolite concentrations, fluxes and free energies imply efficient enzyme usage, Nature Chemical Biology volume 12, pages482-489(2016)

36. Perry, S.W., Norman, J.P., Barbieri, J., Brown, E.B., Gelbard, H.A., Mitochondrial membrane potential probes and the proton gradient: a practical usage guide, Biotechniques. 2011 Feb; 50(2): 98-115

37. Saks V.A., Veksler, V.I., Kuznetsov, A.V., Kay, L., Sikk, P., Tiivel, T., Tranqui, L., Oliveres, J., Winkler, K., Wiedemann, F., Kunz, W.S., Permeabilized cell and skinned fiber techniques in studies of mitochondrial function in vivo. Mol Cell Biochem. 1998; 184:81-100

38. Salway, J.G., Metabolism at a Glance, 4th Edition, ISBN: 978-0-470-67471-0, February 2017, WileyBlackwell

39. Sanchez, T., Wang, T., Venturas, M., Zhang, M., Esencan, E., Sakkas, D., Needleman, D., Seli, E., Metabolic imaging with the use of fluorescence lifetime imaging microscopy (FLIM) accurately detects mitochondrial dysfunction in mouse oocytes. Fertility and Sterility, 2018, 110, 7

40. Sanchez T., Venturas, M., Aghvami, S.A., Yang, X., Fraden, S., Sakkas, D., Needleman, D.J., Combined noninvasive metabolic and spindle imaging as potential tools for embryo and oocyte assessment. Human Reproduction 2019, 1, 13 
41. Shaw J. M., and Nunnari, J., Mitochondrial dynamics and division in budding yeast, Trends Cell Biol. 2002 Apr; 12(4): 178-184.

42. Sharick, J.T., Favreau, P.F., Gillette, A.A., Sdao, S.M., Merrins, M.J., Skala, M.C., Protein-bound NAD $(P) H$ Lifetime is Sensitive to Multiple Fates of Glucose Carbon, Sci Rep., 2018, 8:5456

43. Skala, M.C., Riching, K.M., Bird, D.K., Gendron-Fitzpatrick, A., Eickhoff, J., Eliceiri, K.W., Leely, P.J., Ramanujam, N., In vivo Multiphoton Fluorescence Lifetime Imaging of Protein-bound and Free NADH in Normal and Pre-cancerous Epithelia. J Biomed Opt. 2007 Mar-Apr; 12(2): 024014

44. Takhaveev, V., Heinemann, M., Metabolic heterogeneity in clonal microbial populations. Current opinion in microbiology, 2018 Oct; 45:30-38

45. Wallace, D.C., Mitochondria and cancer, Nat Rev Cancer. 2012 Oct; 12(10): 685-698

46. Wiechert, W., 13C Metabolic flux analysis, Metabolic Engineering, 2001, 3(3), 195-206

47. Westermann and Neupert, Mitochondria-targeted green fluorescent proteins: convenient tools for the study of organelle biogenesis in Saccharomyces cerevisiae

48. Yellen G., Fueling thought: Management of glycolysis and oxidative phosphorylation in neuronal metabolism. J Cell Biol. 2018 Jul 2;217(7):2235-2246.

\section{Acknowledgements}

We thank Carlos Manlio Díaz-García, Gary Yellen, Peter Foster, Easun Arunachalam, Yu-Chen Chao, Bill Ireland and Denis Titov for suggestions, advice, and comments on the manuscript. This work is supported by the National Institute of Health (R01HD092550-01) and the National Science Foundation (PFI-TT1827309, PHY-2013874, MCB-2052305). X.Y. and D.J.N. acknowledge discussions with participants of the "Cellenergy19" and the "Active20" KITP programs, supported in part by the National Science Foundation Grant No. NSF PHY-1748958, NIH Grant No. R25GM067110, and the Gordon and Betty Moore Foundation Grant No. 2919.02.

\section{Author contributions}

X.Y. and D.J.N. conceptualized the work. X.Y. performed the experiments, modeling and data analysis. X.Y. and D.J.N. wrote the manuscript. 